\title{
COMPUTATIONAL MODELING OF A CELL-BASED MICROPHYSIOMETER
}

\author{
By \\ Sebastian J. Eluvathingal \\ Thesis \\ Submitted to the Faculty of the \\ Graduate School of Vanderbilt University \\ in partial fulfillment of the requirements \\ for the degree of \\ MASTER OF SCIENCE \\ in \\ Mechanical Engineering
}

December, 2005

Nashville, Tennessee

Approved:

Professor Mark A. Stremler

Professor Greg Walker

Professor G. Kane Jennings 


\section{ACKNOWLEDGEMENTS}

I would like to thank my advisor Professor Mark A. Stremler for his unwavering support, guidance and patience, even when things looked bleak, throughout my studies at Vanderbilt University.

Gratitude also goes to Professor Wikswo and his research team at VIIBRE for all the help obtained during this work. For the experimental data I would like to thank Dr. Sven Eklund and Professor Cliffel's research group. The funding from Defence Advanced Research Projects Agency (DARPA) for the microphysiometer research is also much appreciated.

Thanks is also due to Dr. Narcisse A. N'Dri for all his timely inputs and trouble shooting expertise and to all my colleagues in the fluid mechanics laboratory for making it such a fun place to work in

I would also like to thank my parents and sister who have taught me a great deal in life and supported me in all my endeavors. To all my roommates and friends, from both $1 \mathrm{~A}$ and $4 \mathrm{~K}$ (You know who you are!!), a big thank you for making life so much more pleasant. Finally I would like to thank Ramya Balachandran and Vishal Koparde for their support and patience while proof reading my thesis. 
ACKNOWLEDGEMENTS .......................................................................... ii

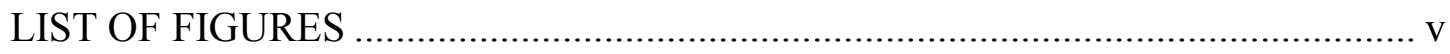

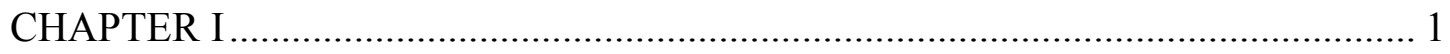

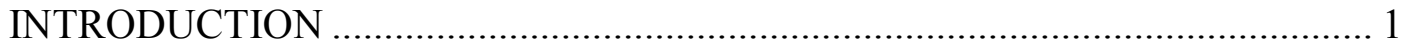

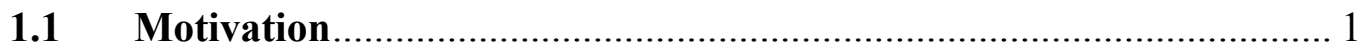

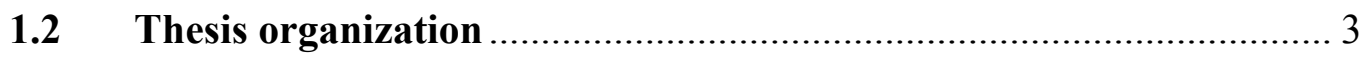

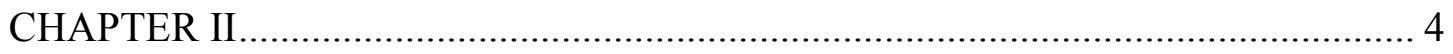

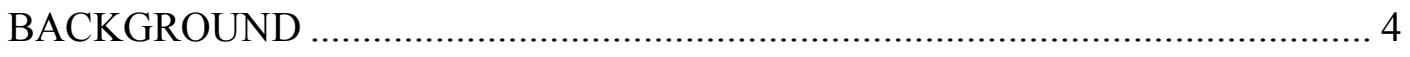

2.1 Cellular metabolism ........................................................................ 4

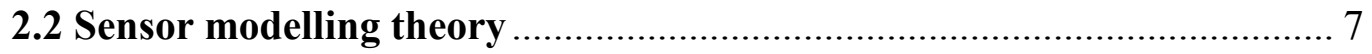

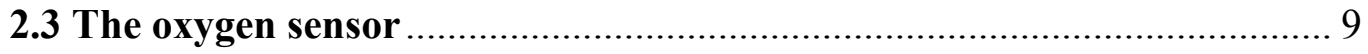

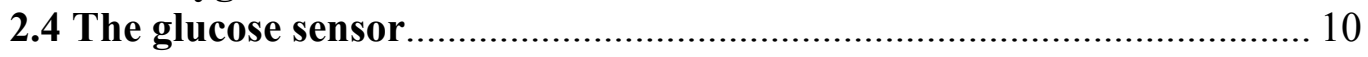

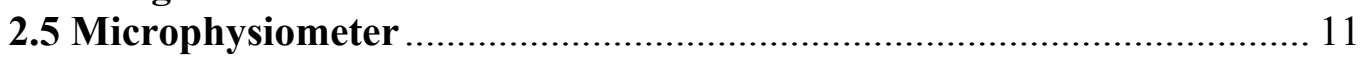

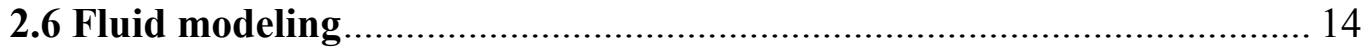

2.9 Computational fluid dynamic modeling using CFDACE \& FLUENT .. 18

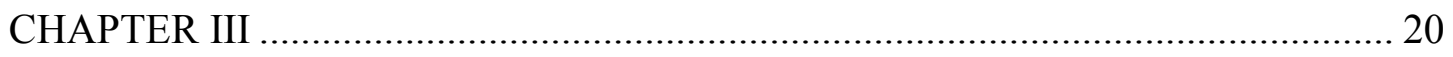

MICROPHYSIOMETER BOUNDARY CONDITONS, GEOMETRY \&

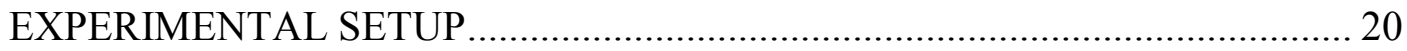

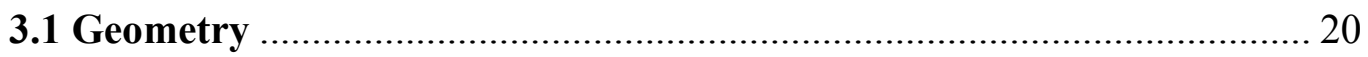

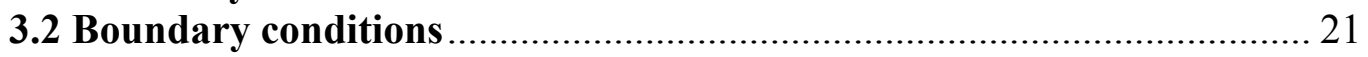

3.2 3D Microphysiometer modeling with CFDACE …............................ 23

3.3 Microphysiometer geometry for oxygen sensing with FLUENT ........... 26

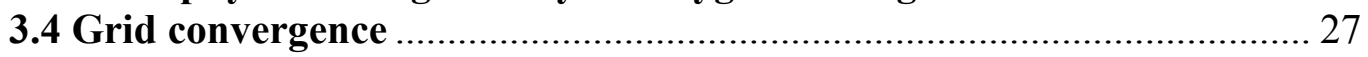

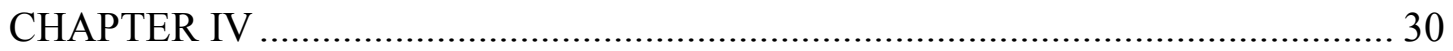

OVERVIEW OF RESULTS ...................................................................... 30

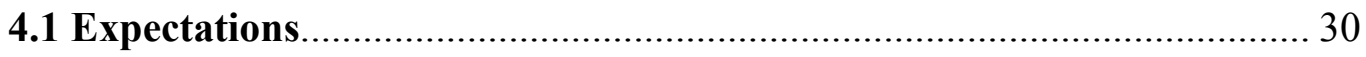

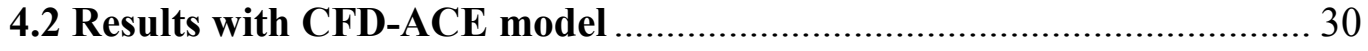

4.5 3D model comparisons with experimental data .................................. 44 


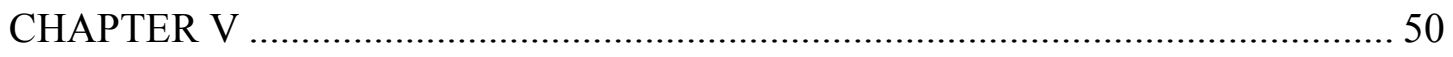

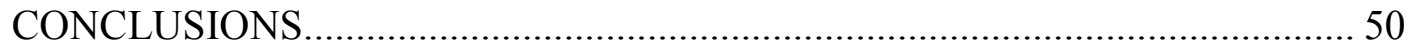

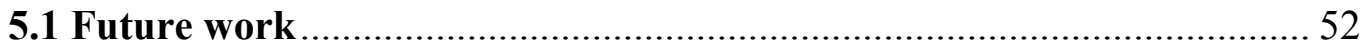

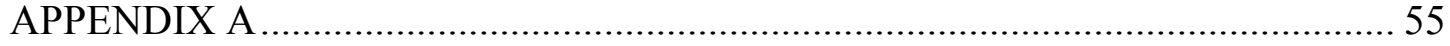

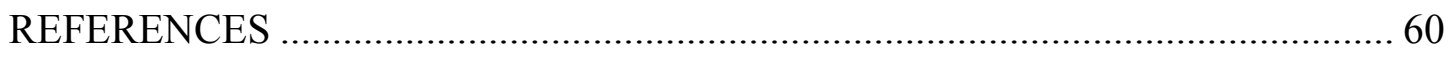




\section{LIST OF FIGURES}

Figure

Page

Figure 1: General metabolic pathways and cycles in living cells (Bruce et al. 1998).. 6

Figure 2: Schematic of a biosensor (Mohanty et al. 2001)....

Figure 3: Schematic diagram of an electrochemical enzyme sensor: A) electrode B) enzyme layer, C) membrane D) solution (Adrian W. Bott et al. 1998).

Figure 4: Schematic of Glucose sensor along with modifications used to model the volume reaction by the addition of a volume to the surface of the original sensor

Figure 5: Cross-sectional schematic of a Microphysiometer (User's manual, Molecular Device Corporation)

Figure 6: Basic geometry of the microphysiometer chamber....

Figure 7: 3D microphysiometer model with boundary conditions explained. 24

Figure 8: 3D CFDACE microphysiometer model showing the grid used 24

Figure 9: Meshed complex microphysiometer model 25

Figure 10: Top view of the 3D microphysiometer modeled using FLUENT.

Streamlines and oxygen concentration profile are shown 26

Figure 11: Side view of the 3D microphysiometer modeled using FLUENT showing streamlines and oxygen concentration profile.

Figure 12: Results for CFDACE model with 72,000 and 150,000 nodes steady state solution with initial inlet concentration $=0.24 \mathrm{mM}$ and cell uptake rate $=-0.00025$ $\mathrm{mM} / \mathrm{secm} 2$

Figure 14: Results for FLUENT model with 500,000 nodes and 1,500,0000 nodes for steady state solution with initial concentration $=0.24 \mathrm{mM}$ and cell uptake rate $=-$ $0.00025 \mathrm{mM} / \mathrm{secm}^{2}$.

Figure 15: Shows first and second order gradient calculation methods 31

Figure 16: Dependence of the sensor response on the initial $\mathrm{O}_{2}$ concentration c, given in $\mathrm{mM}$. Gradients are calculated using the first order approximation 
Figure 17: Dependence of the sensor response on the initial $\mathrm{O} 2$ concentration $\mathrm{C}$ in $\mathrm{mM}$. Gradients are calculated using the first-order approximation

Figure 18: Comparison of First order and Second order approximation of the gradient

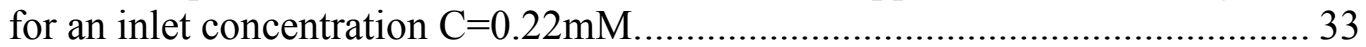

Figure 19: Relative difference between first order vs Second order approximation for an inlet concentration $\mathrm{C}=.22 \mathrm{mM}$.

Figure 20: Parametric study of cell flux uptake (qw in $\mathrm{mM} / \mathrm{secm}$ 2) Current vs Time (cells + Sensor) Second order and $\mathrm{C}=.24 \mathrm{mM}$

Figure 21: Comparing cells on and cells off for qw $=-.00025 \mathrm{mM} / \mathrm{secm}^{2} \& \mathrm{C}=.24 \mathrm{mM}$ for First and Second Order Graph shows Current (cells+sensor) vs Time......... 36

Figure 22: Effect of cells alone for different inlet concentration (C in $\mathrm{mM})$ and cell uptake rates (qw in $\mathrm{mM} / \mathrm{secm}^{2}$ ). Graph shows Current vs Time.

Figure 23: Time averaged versus cell uptake rates $\left(\mathrm{qw}\right.$ in $\left.\mathrm{mM} / \mathrm{secm}^{2}\right)$ for various inlet concentrations $(\mathrm{C}$ in $\mathrm{mM})$ 38

Figure 24: Current (cells off- cells on) vs Time for different Cell uptakes (qw in $\left.\mathrm{mM} / \mathrm{secm}^{2}\right)$ and $\mathrm{C}=.24 \mathrm{mM}$.

Figure 25: Linear relation between coefficients of $x^{3}$ (a) vs cell uptake 40

Figure 26: Linear relation between coefficients of $x^{2}(b)$ vs cell uptake 40

Figure 27: Linear relation between coefficients of $\mathrm{x}$ (c) versus cell uptake................ 41

Figure 28: Linear relation between Constant coefficients $d$ versus cell uptake 41

Figure 29: Shows 3D model microphysiometer data modeled in FLUENT for steady state solutions with varying qw. A) $\mathrm{qw}=2.5 \mathrm{E}-5 \mathrm{mM} / \mathrm{secm}^{2} \mathrm{~B}$ ) $\mathrm{qw}=1.5 \mathrm{E}-$ $5 \mathrm{mM} / \mathrm{secm}^{2} \mathrm{C}$ ) $\left.\mathrm{qw}=1 \mathrm{E}-5 \mathrm{mM} / \mathrm{secm}^{2} \mathrm{D}\right) \mathrm{qw}=2 \mathrm{E}-5 \mathrm{mM} / \mathrm{secm}^{2}$

Figure 30: Comparing experimental and Modeled data with current on $\mathrm{Y}$ axis and Time on $\mathrm{X}$ axis for $\mathrm{C}=0.24 \mathrm{mM}$ and $\mathrm{qw}=-0.00025 \mathrm{mM} / \mathrm{secm}^{2}$

Figure 31: Current versus Time plots, comparing modeled data with different base currents for two different sets of data $\mathrm{C}=0.24 \mathrm{mM} \mathrm{\&} \mathrm{qw}=-0.00025 \mathrm{mM} / \mathrm{secm}^{2} .46$

Figure 32: Comparison of experimental data and modeled data for concentration $\mathrm{C}=0.24 \mathrm{mM}$ at base current $49.5 \%$ base current with different exponential inlet boundary conditions 
Figure 33: Current versus Time plots showing the effects of varying the initial concentrations $(\mathrm{C}$ in $\mathrm{mM})$ with current versus time .............................................. 48

Figure 34: Current versus Time plots showing the effects of Cell uptakes (qw in $\mathrm{mM} / \mathrm{secm}^{2}$ ). 


\section{CHAPTER I}

\section{INTRODUCTION}

\subsection{Motivation}

Researchers are striving to use cell-based biosensors in the fight against novel chemical or biological agents. These cell-based biosensors will be used to detect and analyze such agents (Fraser 1994,1995). When a new biological agent is found, the cell based biosensors will be used to determine the effect of this new agent on a group of cells by studying the consumption or production of some important analytes by the cells in real time. The volume of the device is in the order of micro liters and it uses cell metabolism as a method of detection.

A collaborative effort between the Vanderbilt Institute for Integrative Biosystems Research and Education and the Vanderbilt Medical Center has modified a commercially available microphysiometer (Kruger 2002) or cytosensor (Molecular Devices Incorporated California) so that it can detect up to five important analytes. This modified microphysiometer provides a powerful cell based biosensor (Eklund et al. 2004). This fluid based device was developed to monitor the metabolism of living cells in real time (Buerk 1993). Different toxic agents can affect and even block specific metabolic pathways of the cells (Verhaegen 2000) (Figure 1). Since different toxic agents affect different metabolic pathways, the toxic agents can be categorized and identified by monitoring the cells metabolic response (Verhaegen 2000) (Wu et al. 2001). The microphysiometer will be used to see the effect of such chemical or 
biological agents on a group of cells by measuring five important analytes simultaneously (Figure 5). The ultimate aim is to simultaneously use a number of microphysiometers, with each containing a different type of cell (such as heart or lung) to study the effects of the biological agent on each different cell type.

Continued improvements in the computing speeds of computers have now made it possible to model very complex systems, from airplanes to biological organs (Abbot 1989). In this thesis, commercially available Computational Fluid Dynamics (CFD) tools are used to model and analyze the microphysiometer. This method will reduce the need for a large number of trial and error prototypes; thereby saving a huge amount of resources, as fabricating these devices is quite expensive both in terms of time and money.

A number of parameters need to be studied in the computational model. The experimental team does not have an exact value for the cell analyte uptake as this is very hard to measure accurately. A study of the sensor output of various analyte uptakes by the model will be done and then predictions will be made of the cell uptake based on the sensor output using mathematical models. Another problem faced by the experimental group is that since the microphysiometer is a very small device the currents produced are also very small. It is very difficult to check if the current being produced is just noise or whether it actually makes sense. The CFD modeling will look at the ideal situation and will be able to assess if the current produced makes sense or not. This would also enable the tweaking of certain parameters in such a way that the output current is enhanced or increased in magnitude. Response time for the effects of the cells on the sensor will also be studied. The effect of all the other 
different parameters will also be seen on the sensor output. The final objective is to come up with a mathematical model that can match the computational model to give the model output with good accuracy for any initial concentration of analytes in the microphysiometer as well as for any cell analyte uptake.

\subsection{Thesis organization}

Chapter 2 of this thesis covers the background, of this work including a literature review, an introduction to sensor modeling theory, an overview of the fluid dynamics theory involved in this model and the experimental methods used to run the microphysimeter. Chapter 3 presents the geometries used in modeling the microphysiometer and establishes the computational domains for these geometries. The various modifications that have been incorporated into the geometric model are also studied. This chapter will also cover parameters used in modeling the microphysiometer including boundary volume and initial conditions. Chapter 4 analyzes the findings of the model and suggests refinements to the model. This chapter also compares the microphysiometer performance with cells and without cells and studies the effect of the operating parameters on magnitude of output current and base line current. Parametric analysis of the initial concentration $(\mathbf{C})$ and cell uptake rates (qw) and optimization will also be discussed. Chapter 5 contains the results and conclusions of the thesis. 


\section{CHAPTER II}

\section{BACKGROUND}

This chapter introduces the microphysiometer, including its design principles, geometry, and chemistry. It also presents the background of cell metabolism, the convection diffusion equations and how electrochemical sensors observe changes in a large number of metabolites as they change with time.

\subsection{Cellular metabolism}

Cellular metabolism is the process by which cells take in nutrients such as carbohydrates (glucose) and oxygen and convert these nutrients into energy and waste material. The waste materials are usually acids such as carbonic acid and lactic acid. It has been determined experimentally that there is a very close relationship between the rates at which these nutrients are converted and the rates at which these acids are created. So by studying the acids we can monitor the nutrients themselves. Cell metabolism consists of a large number of metabolic cycles. In the cells of mammals these include the three primary aerobic cycles shown in figure 1 which are the phosphate cycle, the glycolysis cycle and the citric acid cycle (Becker et al. 1996). In the glycolysis cycle, glucose and glutamine are converted to pyruvate based on the concentrations. Depending on the concentrations and reaction rates, part of the

glucose is also converted to lactate. The other two cycles involve the conversion of oxygen to water and waste products with the help of Adenosine tri-phosphate (ATP)( 
Whiteley 2001). The main waste products are lactic acid and carbonic acid. In this thesis we will be studying the effect of only two of the five metabolites that the microphysiometer measrures, oxygen and glucose which take part in the phosphate and glycolysis cycles. 


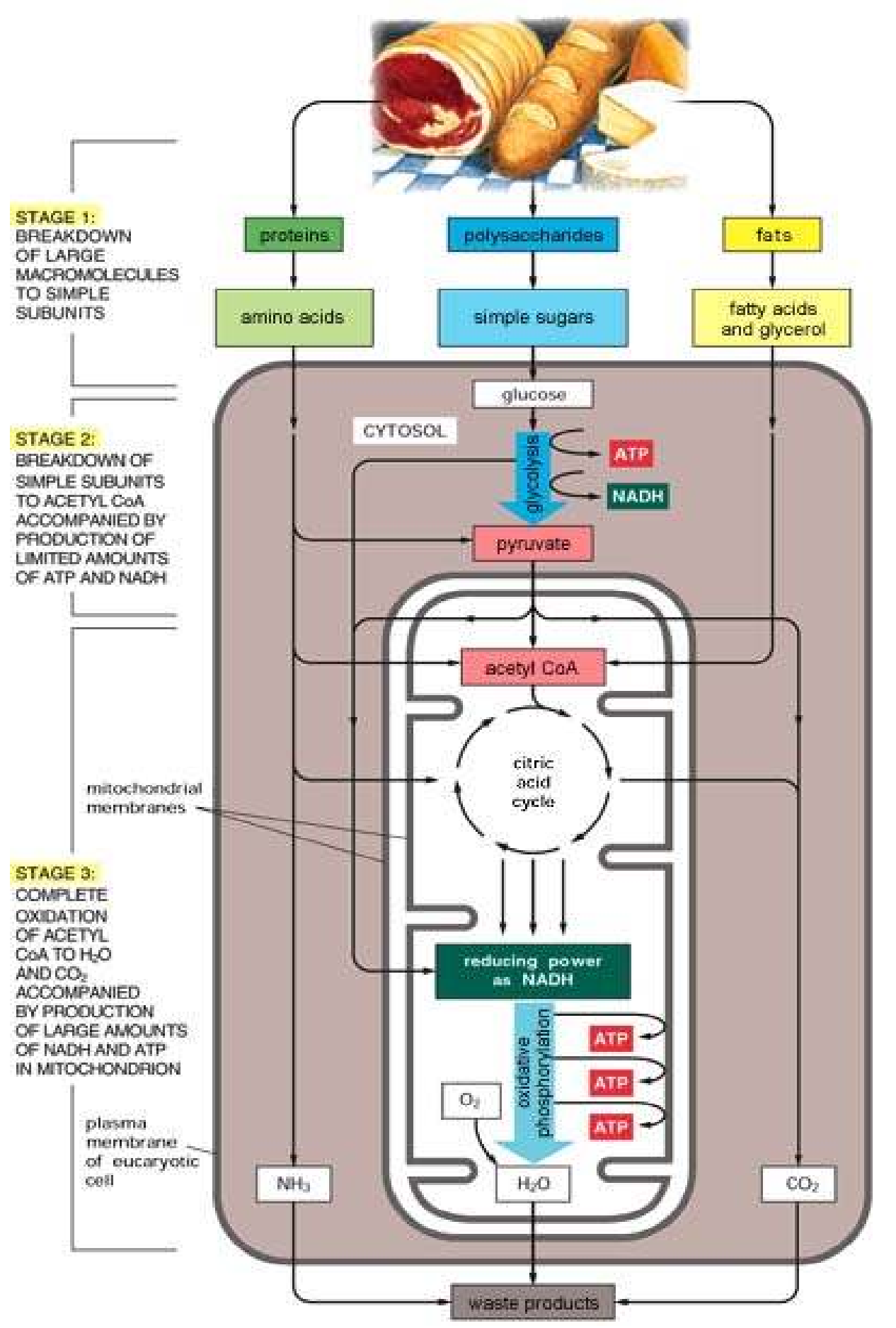

Figure 1: General metabolic pathways and cycles in living cells (Bruce et al. 1998) 


\subsection{Sensor modelling theory}

A sensor is broadly defined as a device that responds to a stimulus, such as heat, light, or pressure, and generates a signal that can be measured or interpreted. In the microphysiometer, the sensor generates an electrical signal that is driven by the concentration gradient of the analytes very close to the sensor (Higson et al. 1994).

The microphysiometer is an electrochemical biosensor. It uses fluid solution as the medium to carry the analytes and the foreign chemical species or toxins. This solution has very similar properties to water. The term biosensor has been applied to devices that use biological substances such as enzymes, antibodies, and nucleic acids as bio-recognition elements. These two types of sensors are extremely well known for their high sensitivity, small size, low cost, versatility and stand alone operation. The biosensor consists of primarily two parts as shown in figure 2: the receptor and the detector (Mohanty et al. 2001). The receptor includes the bio-recognition elements, which recognize the substance of interest, and the transducer, which converts the biorecognition event into a measurable signal. The detector consists of the signal processing system, which converts the signal into a usable form.

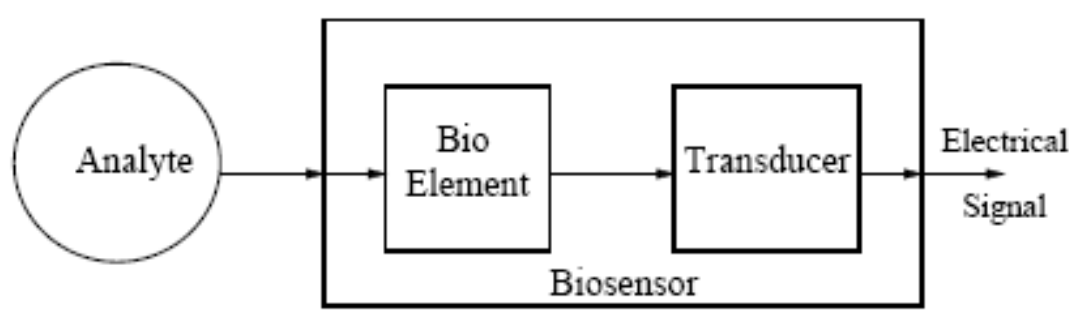

Figure 2: Schematic of a biosensor (Mohanty et al. 2001) 
Cell-based biosensors use living biological cells to sense changes in the extracellular environment that (hopefully) have been caused by physiological changes in the cell that are the result of the presence of toxic agents and pathogens. Biosensors are typically small and easily transportable devices that use extracellular recordings and optical measurements to detect the physiological changes. The biggest application of biosensors is in the field of broad range detectors where they are used to find and assess unknown threat agents (Eklund, et al. 2004).

Cell based biosensors can be classified as two basic types (Mohanty, et al. 2001), electrical detection biosensors and cell metabolism detection sensors. Electrical detection biosensors, use electrochemical methods for transduction. These can be subdivided into two basic types. The first type, potentiometric sensors, measure the electrochemical potential of a cell at zero current. The potential will be proportional to the logarithm of the concentration of the substrate being measured. The acidification rate in the microphysiometer is measured using this method. This thesis work does not include this sensor. The second type is the amperometric sensors, which applies an increasing or decreasing potential to the cell. Oxidation or reduction of the substance to be analyzed occurs (Kissinger, 1997), which results in a sharp change in the cell current to give an output current. The current generated is directly proportional to the gradient of concentration of the electroactive species. The oxygen sensor modeled in the present work for the microphysiometer is of this kind. The second type of biosensor is the cell metabolic detection sensor. These enzymatic biosensors are based on the biospecificity of an enzymatic reaction and an electrode reaction. The enzymatic oxidation of glucose produces hydrogen peroxide, which in 
turn generates electrons by electrode reaction (Cunningham, 1998; Burns, 2000; Aravanis, 2001). The current density is used as a measure of glucose in the sample. It rates an electric current or a potential difference for quantitative analysis. The glucose sensor modeled in the present work for the microphysiometer is of this kind.

A combination of the above two microfluidic based sensors are used in the cell based biosensor (microphysiometer) currently being studied in Vanderbilt. The microphysiometer uses five different electrochemical sensors to measure the five analytes. The two electrochemical sensors modeled in this thesis are the oxygen sensor and the glucose sensor.

\subsection{The oxygen sensor}

The oxygen sensor was first revolutionized in 1950's by the American scientist Leland C. Clark. He studied the electrochemistry of oxygen gas reduction at platinum metal electrodes, pioneering the creation of the "Clark electrodes". The Clark oxygen electrodes that were invented in 1954 still remain the standard for measuring the concentration of dissolved oxygen in biomedical, environmental and industrial applications.

Clark sealed the already available platinum electrode in glass and covered it with cellophane. He then experimented with various membranes until he constructed the first membrane covered oxygen electrode having both the anode and the cathode behind a nonconductive polyethylene membrane. The electrodes are separated from the sample by a semi-permeable membrane that provides the mechanism to diffuse oxygen into the sensor (Clark, L.C. Jnr. Ann. NY Acad. Sci. 102, 29-45, 1962). These 
membranes have limited permeability for oxygen and hence reduce depletion of oxygen from the sample thereby making it possible to have quantitative measurements of oxygen concentration (Astrup1986). The electrical current resulting from this limited permeability of oxygen is directly proportional to the oxygen concentration in the sample.

\subsection{The glucose sensor}

Clark and Lyons first coined the term 'enzyme electrode' (Clark, L.C. Jnr. Ann. NY Acad. Sci. 102, 29-45, 1962). Clark then converted these ideas to measure glucose as well $\mathrm{O}_{2}$. The most successful commercial biosensors are amperometric glucose biosensors. In a glucose sensor, an enzyme acts as a bio-recognition element, which recognizes glucose molecules (Figure 3 and 4). These enzyme molecules are located on an electrode surface, which acts as a transducer. As soon as the enzyme recognizes the glucose molecules, it acts as a catalyst to produce gluconic acid and hydrogen peroxide from glucose. The electrodes consume the electrons transferred due to hydrogen-peroxide oxygen coupling. The resulting electron flow is proportional to the number of glucose molecules present in the fluid solution. 


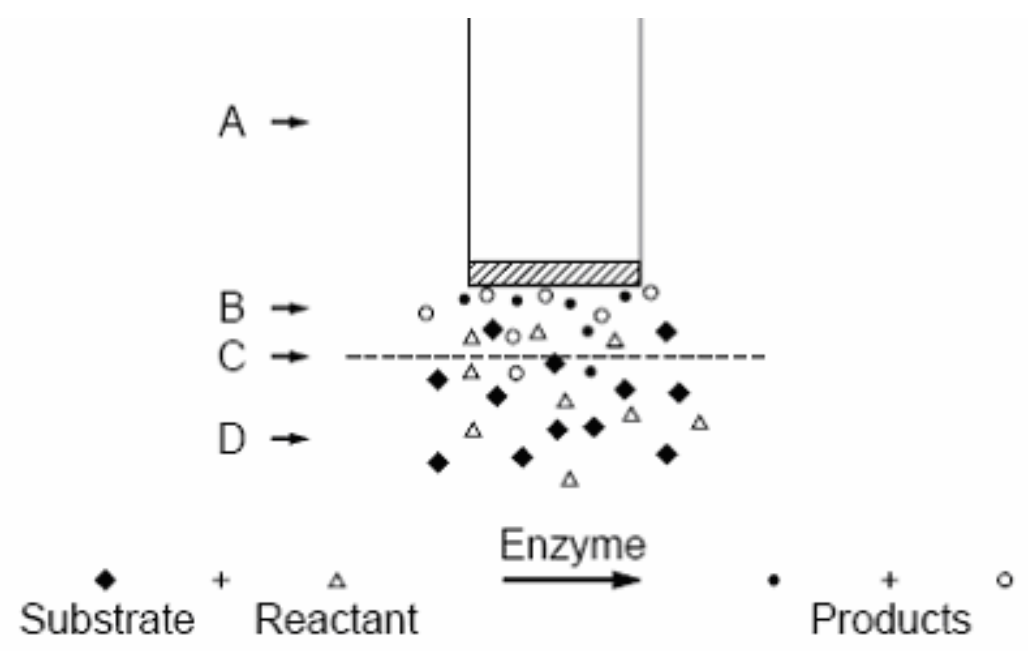

Figure 3: Schematic diagram of an electrochemical enzyme sensor: A) electrode B) enzyme layer, C) membrane D) solution (Adrian W. Bott et al. 1998)

\subsection{Microphysiometer}

The microphysiometer has been designed to monitor the metabolic changes which affect the rate at which analytes are produced or consumed by the cells. These responses can be transient or steady. Using this method, the microphysiometer can provide a real time non invasive means of measuring cellular responses to a wide variety of agents.

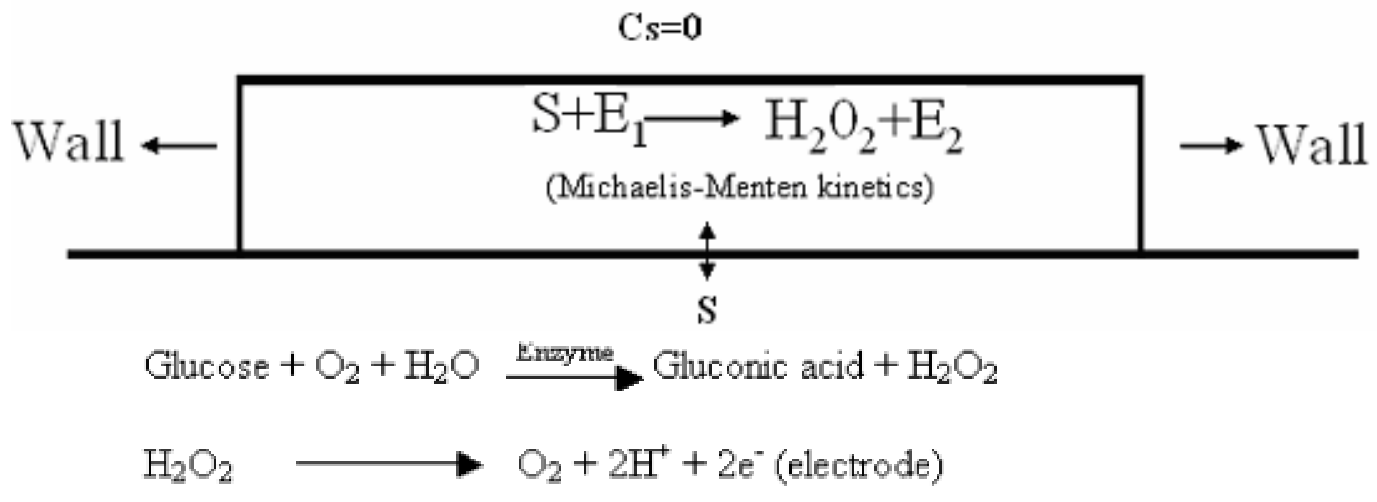

Figure 4: Schematic of Glucose sensor along with modifications used to model the volume reaction by the addition of a volume to the surface of the original sensor 
Five different analytes were chosen to be the parameters that will be measured by the microphysiometer in order to study the cells metabolism. These analytes are oxygen, glucose, carbon dioxide, lactate, $\mathrm{pH}$ and nicotinamide adenine dinucleotide (NADH). A basic cross-sectional diagram of the microphysiometer is shown in Figure 5. The cells are placed at the bottom of the sensor chamber. The plunger and the sensor chamber walls form a flow chamber above the cells in the capsule. The microphysiometer delivers fluid to the cells through the fluid inlet.

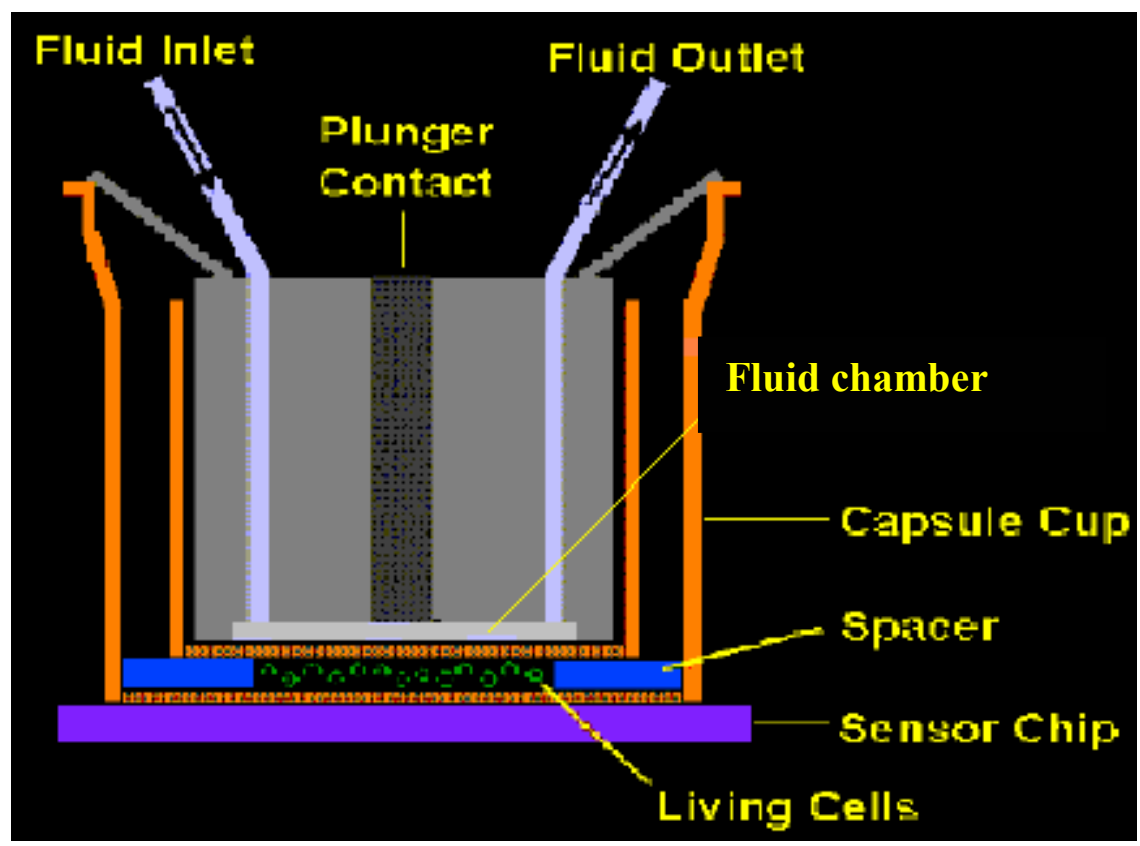

Figure 5: Cross-sectional schematic of a Microphysiometer (User's manual, Molecular Device Corporation)

There are two electrical contacts one between the microphysiometer and the fluid in the sensor chamber and the other between the microphysiometer and the back of the sensor chip. As long as the medium flows through the sensor chamber, there is no buildup of metabolites and the concentration of analytes in the chamber remains steady at approximately the concentration of the medium. The concentration is 
measured when the flow is off. The steady state solution depends on the concentration of the fresh medium, its flow rate, and the rate at which the cells are excreting the analytes. When the pump is stopped, acidic metabolites begin to build up in the cell chamber and the $\mathrm{pH}$ begins to drop. Since the cell volume is very small even a minute change in metabolites causes a detectable change in the chamber's concentration gradients. This is also the case with oxygen concentration. As the flow of the pump is stopped the oxygen concentration comes down because the cells continuing consuming the oxygen. Even a small decrease in the oxygen levels in the sensor volume is noticeable as the total volume is very small.

The experimental consists of two steps. The first step is a flushing cycle, which is the flow cycle with inlet turned on for about 35 seconds. This is modeled as a steady state solution. The sensing cycle is the second step. It lasts for 30 seconds. Here the flow in switched off. This is the transient part of the procedure. The oxygen levels are obtained using the current at the surface of the electrode sensor. This measured current is directly proportional to gradient of concentration at the surface of the electrode sensor according to (Fick, A. 1885).

$$
\mathrm{I}=\mathrm{F} \times \mathrm{A} \times \mathrm{D} \times\left(\frac{\partial \mathrm{C}}{\partial N}\right)
$$

where $\mathrm{I}$ is the current and $\mathrm{D}=$ Diffusivity of the analyte $\left(2.009 * 10^{9} \mathrm{~m}^{2} / \mathrm{sec}\right), \mathrm{F}$ is the Faradays constant $(96485 \mathrm{Cb} / \mathrm{mol})$ and $\mathrm{A}$ is the surface area of the sensor. For glucose the sensor is slightly different. Here a volume reaction following according to Michaelis-Menten kinetics takes place as discussed earlier. The electrode sensor used 
for measuring glucose has a volume reaction taking place in it though the general equation for measuring current is the same as in the oxygen sensor.

\subsection{Fluid modeling}

The governing equations for the flow, which represent mathematical statements of the conservation laws of physics, are the continuity equations and momentum conservation equations. These two conservations laws can be used to represent the current problem using the Navier-Stokes equation, which is solved computationally. The conservation of mass, or continuity equation, requires that the time rate of change of mass in a control volume be balanced by the net mass flow into the same control volume.

$$
\frac{\partial \rho}{\partial t}+\nabla \cdot(\rho \mathbf{u})=0
$$

In this thesis flow is assumed to be incompressible flow, so the density of the fluid solution always remains constant. The motion of the fluid is also governed by the Navier-Stokes equations. The Navier-Stokes equations (White 1994) are formed by combining the fluid kinematics and constitutive relations into the fluid equation of motion and eliminating the strain rate and fluid stress parameters. These equations are basically the differential forms of the linear momentum principle. The assumptions made are that the fluid is Newtonian, continuity is satisfied and the fluid is isotropic.

The $\mathrm{x}$ component of the momentum equations is found by setting the rate of change of the $\mathrm{x}$ momentum of the fluid particle equal to the total force due to the surface stresses in the $\mathrm{x}$ direction of the element. In Newtonian flows the viscous stresses are proportional to the deformation rates. The nine viscous stress components 
are proportional to the deformation rates and can be related to the velocity gradients to produce the shear stress terms. Substituting the shear stress terms into the momentum equations yields

$$
\begin{aligned}
& \rho\left(\frac{\partial v_{x}}{\partial t}+v_{x} \frac{\partial v_{x}}{\partial x}+v_{y} \frac{\partial v_{x}}{\partial y}+v_{z} \frac{\partial v_{x}}{\partial z}\right)=\mu\left[\frac{\partial^{2} v_{x}}{\partial x^{2}}+\frac{\partial^{2} v_{x}}{\partial y^{2}}+\frac{\partial^{2} v_{x}}{\partial z^{2}}\right]-\frac{\partial p}{\partial x}+\rho g_{x} \\
& \rho\left(\frac{\partial v_{y}}{\partial t}+v_{x} \frac{\partial v_{y}}{\partial x}+v_{y} \frac{\partial v_{y}}{\partial y}+v_{z} \frac{\partial v_{y}}{\partial z}\right)=\mu\left[\frac{\partial^{2} v_{y}}{\partial x^{2}}+\frac{\partial^{2} v_{y}}{\partial y^{2}}+\frac{\partial^{2} v_{y}}{\partial z^{2}}\right]-\frac{\partial p}{\partial y}+\rho g_{y} \\
& \rho\left(\frac{\partial v_{z}}{\partial t}+v_{x} \frac{\partial v_{z}}{\partial x}+v_{y} \frac{\partial v_{z}}{\partial y}+v_{z} \frac{\partial v_{z}}{\partial z}\right)=\mu\left[\frac{\partial^{2} v_{z}}{\partial x^{2}}+\frac{\partial^{2} v_{z}}{\partial y^{2}}+\frac{\partial^{2} v_{z}}{\partial z^{2}}\right]-\frac{\partial p}{\partial z}+\rho g_{z}
\end{aligned}
$$

Passive scalars are used to model the analytes in the flow. Here the passive Passive scalars have 0 mass and behave as tracer particles that do not affect the flow. They are transported convectively in the fluid volumes by the local flow field. In the model the passive scalars are also allowed to move through diffusion.

The convection equations and the diffusion equations together model the transport of passive scalars (Janata 1989). Let $\delta V$ be an infinitesimally small volume of fluid at a position $\vec{x}$. Let the number of analyte molecules in this volume be $\mathrm{N}(\vec{x}, \mathrm{t})$. Then concentration will be defined as

$$
C=\lim _{\delta V \rightarrow 0} \frac{\delta N}{\delta V} .
$$

The units of $\mathrm{C}$ will be in molars. Then the convective flux or mass of the metabolites caused by the convective flow $\mathrm{u}$ is defined as

$$
Q_{\text {Convective }}=u C
$$


The metabolites are also also allowed to diffuse. The diffusive flux is proportional to the concentration gradient according to Ficks first law of diffusion. If $\mathrm{D}$ is the diffusivity of the analyte, then

$$
Q_{\text {Diffusive }}=D \frac{d C}{d n}
$$

The diffusivity is found experimentally using the relationship (2.7)

$$
D=\frac{\left(\frac{K^{3}}{\pi^{2} m}\right)^{\frac{1}{2}} T^{\frac{3}{2}}}{p^{2}}
$$

where $\mathrm{K}$ is the Boltzman constant, $\mathrm{m}$ is the mass of the diffusing species, $\mathrm{p}$ is the pressure and $\mathrm{T}$ is temperature. Fick's second law gives the relation between diffusion and concentration with respect to time(t) as

$$
\frac{d C}{d t}=D \nabla C
$$

The convective flux and the diffusive flux can be combined into the convectiondiffusion equations

$$
\frac{\partial C}{\partial t}+\nabla(u C)=D \Delta C
$$

Since passive scalars are being used, the Navier-Stokes equations can be solved independently, of the convective-diffusion equations Now in general the convection-diffusion equations and the Navier-Stokes equations have to be solved numerically to obtain the solution for the microphysiometer model because the equations are coupled (Alexandrou 2001). The equations at each point are dependent on every other point and hence are usually very difficult to solve analytically. Both sets of equations are nonlinear partial differential equations. The resulting velocity is 
then used in the convection-diffusion equations to determine scalar transport. The two non-dimensional numbers used are the Reynolds number $(\mathrm{Re})$ and the Peclet number. These numbers are used to decide whether the calculations can be done using just the convection-diffusion equations, or just the Navier-Stokes equation or a combination of both

The Reynolds number (Re) gives the ratio of inertial force to viscous force as is,

$$
\mathrm{Re}=\frac{\rho u l}{v}
$$

For the microphysiometer $\rho=996 \mathrm{~kg} / \mathrm{m}^{3}, u$ is the characteristic flow velocity. $l$ is a characteristic length scale and $v$ is the dynamic viscosity of the fluid $\left(0.001 \mathrm{Ns} / \mathrm{m}^{2}\right)$. In the model the Re is about 5 during the steady state part of the solution which is quite small and hence the flow in the microphysiometer is expected to be laminar (George et al. 2001).

The Peclet number, $\mathrm{Pe}=\left(\left(1^{*} \mathrm{u}\right) / \mathrm{D}\right)$ gives the ratio between the convective flux and the diffusive flux in the flow. When the Peclet number is very small the diffusive flux dominates and when the Peclet number is very large the convective flux dominates.

The diffusivity of oxygen in fluid solution at room temperature is $2.09 \mathrm{E}^{-9} \mathrm{~m}^{2} / \mathrm{sec}$ and the convective velocity is 0.08 meters. During steady state operation, the Peclet number is approximately 2400 when using the maximum velocity that will be found in the volume chamber. This means that the maximum velocity mass transport is dominated by convection, but diffusion is not negligible. This justifies the need to use 
both the Navier-Stokes equation and the convection-diffusion equations to compute this problem.

\subsection{Computational fluid dynamic modeling using CFDACE \& FLUENT}

Computational modeling of the microphysiometer was accomplished using the commercially available codes CFD-ACE and FLUENT. For CFD-ACE, computational grids were generated using CFD-GEOM and post processing was done with CFD-VIEW. CFD-ACE, CFD-GEOM and CFD-VIEW are made available by CFDRC Inc. (Huntsville) and licensed by ESI-Software (Paris). Some further testing was also done using other commercial code FLUENT. For FLUENT, computational grids were generated by GAMBIT and post processing was done using TecPlot. GAMBIT and FLUENT are licensed by FLUENT Inc. FLUENT also uses the finite volume discretization method like CFD-ACE along with similar methods of applying boundary and initial conditions which will be discussed further on.

For modeling with CFD-ACE two modules were coupled together. One was the flow module and the other the user-scalar module. The flow module is the main part of the CFD-ACE solver. Activating the flow module implies that both the velocity fields, i.e. that is $\mathrm{x}, \mathrm{y}$ and $\mathrm{z}$ momentum equations and the pressure field equations, are solved. Similarly in FLUENT the user-scalars option was used to model passive scalars

The most fundamental consideration in CFD is how a continuous fluid is modeled in a discretized fashion on a computer. The method consists of discretizing the spatial domain into small cells to form a volume mesh or grid and then appling a 
suitable algorithm to solve the equations of motion. The mesh can be either irregular (for instance consisting of triangles in 2D, or pyramidal solids in 3D) or regular. The distinguishing characteristic of the former is that each cell must be stored separately in memory. If the problem is highly dynamic and occupies a wide range of scales, the grid itself can be dynamically modified in time, as in adaptive mesh refinement methods. The discretization method in "classical" or standard approaches used most often in commercial software and research codes is the finite volume method. The governing equations are solved on discrete control volumes. This integral approach yields a method that is inherently conservative. CFD-ACE and FLUENT use the finite volume method.

In all of these approaches a geometry is defined based on the physical model. The volume is then discretized to form a mesh. The governing equations are then solved iteratively using specified boundary and initial conditions to reach a steady state or transient state solution. 


\section{CHAPTER III}

\section{MICROPHYSIOMETER BOUNDARY CONDITONS, GEOMETRY \& EXPERIMENTAL SETUP}

\subsection{Geometry}

The overall geometry of the microphysiometer fluid chamber as shown in figure 6 is that of a cylinder with a $2500 \mu \mathrm{m}$ diameter and a $65 \mu \mathrm{m}$ height. The inlet and outlet are holes in the top surface of the cylinder. The inlet has a diameter of $78 \mu \mathrm{m}$ and the outlet is twice the diameter of the inlet. Both the inlet and the outlet are offset by the same distance $940 \mu \mathrm{m}$ from the center. The five sensors are all placed on the same surface as the inlet and outlet. Four of the sensors are symmetrically placed and offset by $780 \mu$ meters from the center. The fifth sensor is larger and is at the center of the surface. The living cells are located all along the bottom of the cylinder separated from the chamber by a permeable membrane.

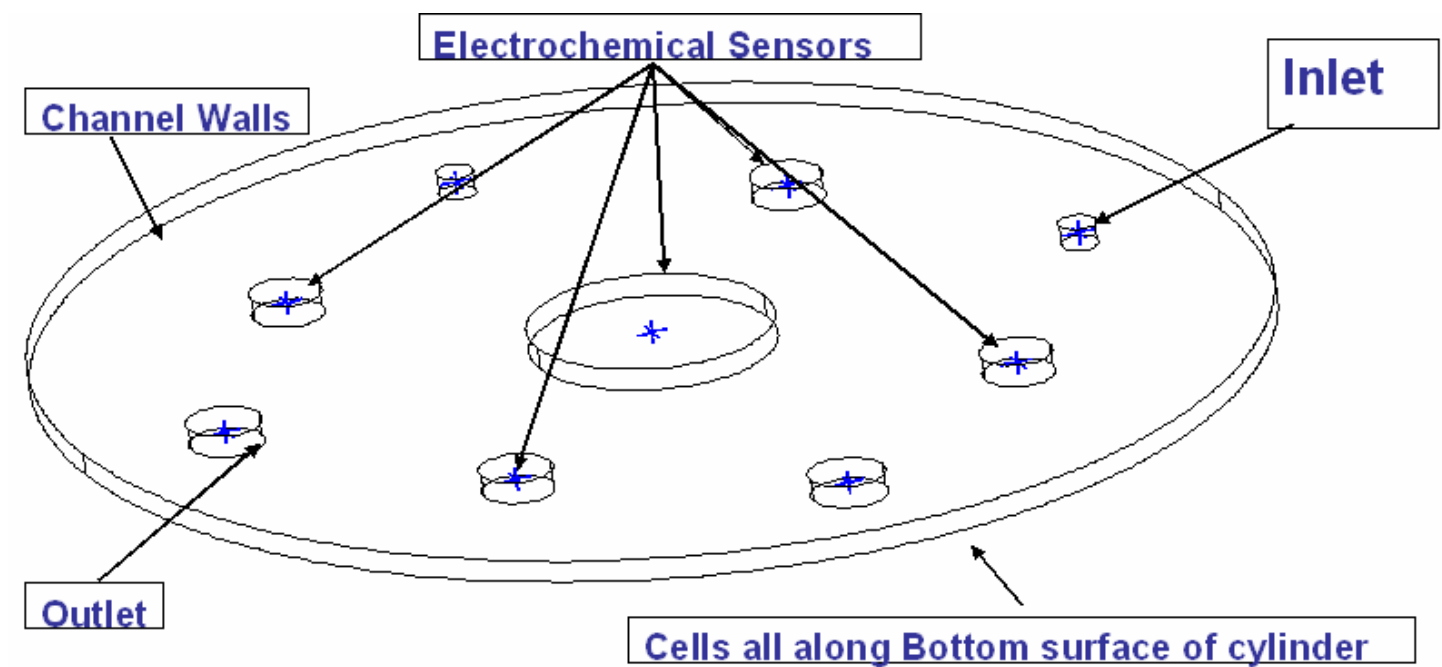

Figure 6: Basic geometry of the microphysiometer chamber 
The inlet is controlled by a valve which is at the end of a long pipe that connects to the micro volume chamber. Similarly the outlet is also connected with the help of a long pipe. The flow is driven by a pump which is turned on during the flushing cycle and turned off during the sensing cycle. The valves do not close instantaneously and take about 1.5 seconds to completely stop the flow.

The experimental procedure consists of 2 parts, a steady state part and a transient state part. During the steady state part of the experiment, a uniform inlet velocity is applied and the model is allowed to run until a steady state solution is reached. In the transient part the uniform inlet velocity is made zero. The data got from the steady state analysis of the flow is used as the initial condition to see the effect of the sensor and the cells. The transient part is run for 30 seconds, which is the duration of the cycle in the experimental method used in running the microphysiometer.

\subsection{Boundary conditions}

The microphysiometer is modeled with the help of a number of boundary conditions to mimic the cell, sensor and chamber walls in the biosensor. The cell boundaries are given a cell flux uptake to model the cell consumption of analytes. The sensor surface boundary condition is set to zero concentration based on ideal sensor theory, which states that an ideal sensor consumes everything it measures. The walls act as physical boundaries for the medium.

The inlet boundary condition is set to the velocity inlet condition, specifying the velocity to match the capacity of the pumps used in the microphysiometer and 
inlet concentration (C) to match the desired inlet concentration conditions. The outlet boundary condition is made a pressure boundary condition and set to atmospheric pressure conditions. The generalized boundary conditions for user scalars can be stated as

$$
A\left(D \frac{\partial c}{\partial n}\right)+B c=c
$$

The concentration gradient is directly proportional to the cell flux (qw). D is the diffusivity of the analyte in the medium at that temperature. Each of the terms in this equation represents concentration units. Each of the terms A, B and C are set differently for each boundary condition.

The terms are set to $\mathrm{A}=0, \mathrm{C}=0$ and $\mathrm{B}=1$ for the sensor boundary condition, which amounts to setting the concentration at the sensor wall to zero.

To obtain the cell wall boundary condition $\mathrm{A}=1, \mathrm{C}=\mathrm{x} \mathrm{mM} / \mathrm{secm}^{2}$ and $\mathrm{B}=0$, which defines a constant cell flux x (qw) for the cell wall boundary condition. This simulates the biological cycles going on in the cells (as described in section 2.1).

For the normal wall boundary condition we set $\mathrm{A}=1, \mathrm{C}=0$ and $\mathrm{B}=0$ which sets $\mathrm{qw}=0 \mathrm{mM} \cdot \mathrm{sec} / \mathrm{m}^{2}$ to define the wall. No-slip boundary condition i.e. the velocity of the medium closest to the boundary equals zero, is also applied to the walls and the sensors.

The sensor boundary condition and the wall boundary condition are the same throughout the current study. 


\subsection{D Microphysiometer modeling with CFDACE}

The three dimensional computational geometry was exactly the same as the microphysiometer chamber described in the beginning of this chapter (Figure 7). The geometrical mesh was created by first meshing a plane surface mesh which has interior circular faces with bidirectional sides within it to model the sensors. This planar surface and mesh was then extruded to obtain the required thickness (Figure 8). The entire lower surface is given the cell diffusion flux boundary condition for cell oxygen or glucose intake. All the vertical walls in the interior of the volume are made permeable interfaces.

This geometry has a choice of 5 sensor positions. For sensing oxygen, the sensors closest to the outlet are used. Again the modeling is done in two parts, the steady state part and the transient state part. The steady part consists of the flushing cycle with the inlet on and the unsteady part consists of the sensing cycle with the inlet turned off. The output of the steady part is used as the input to the unsteady part of the cycle. The Figures 7 and 8 below show both the geometry as well as the mesh characteristics used in the model 


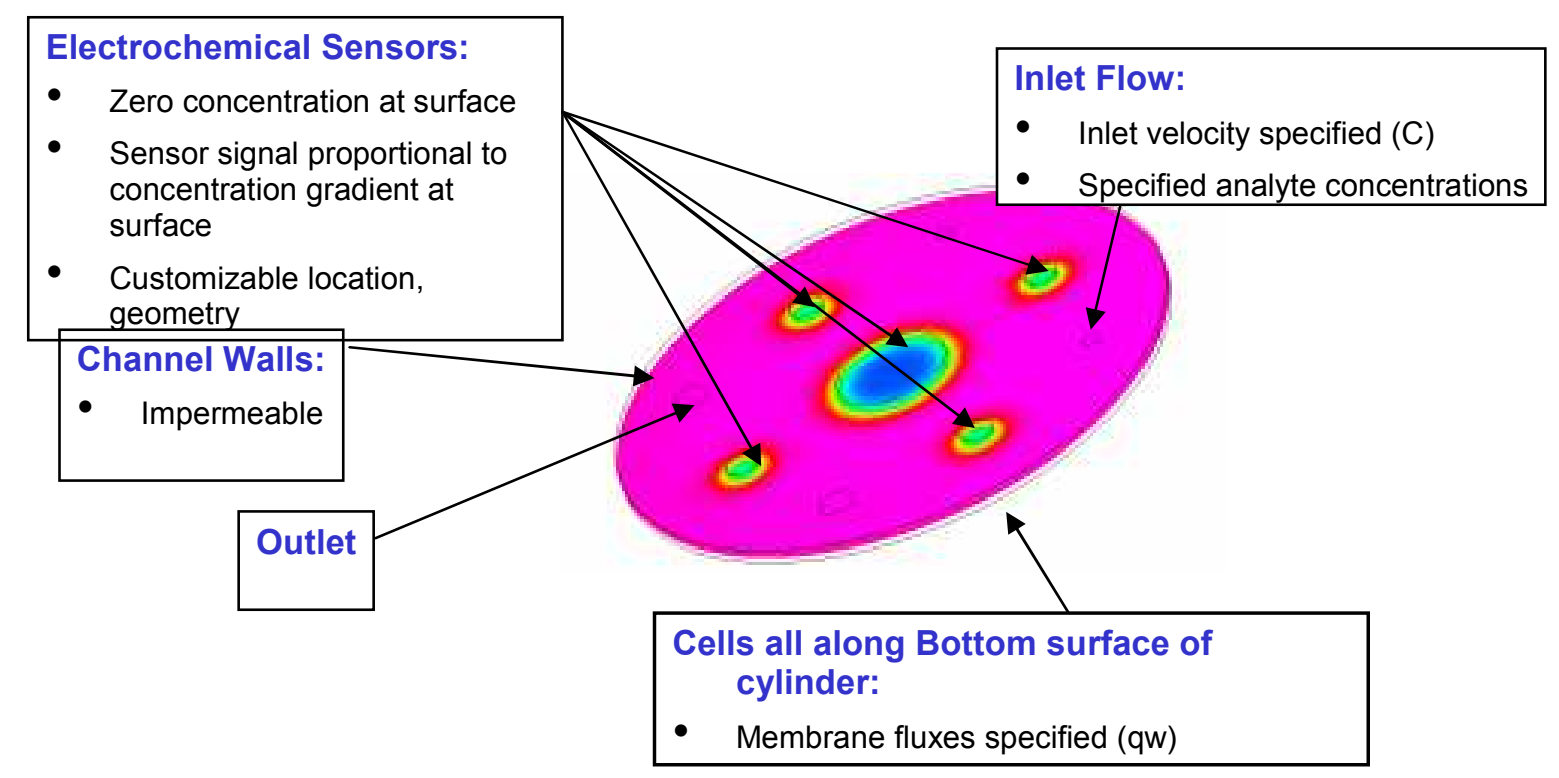

Figure 7: 3D microphysiometer model with boundary conditions explained

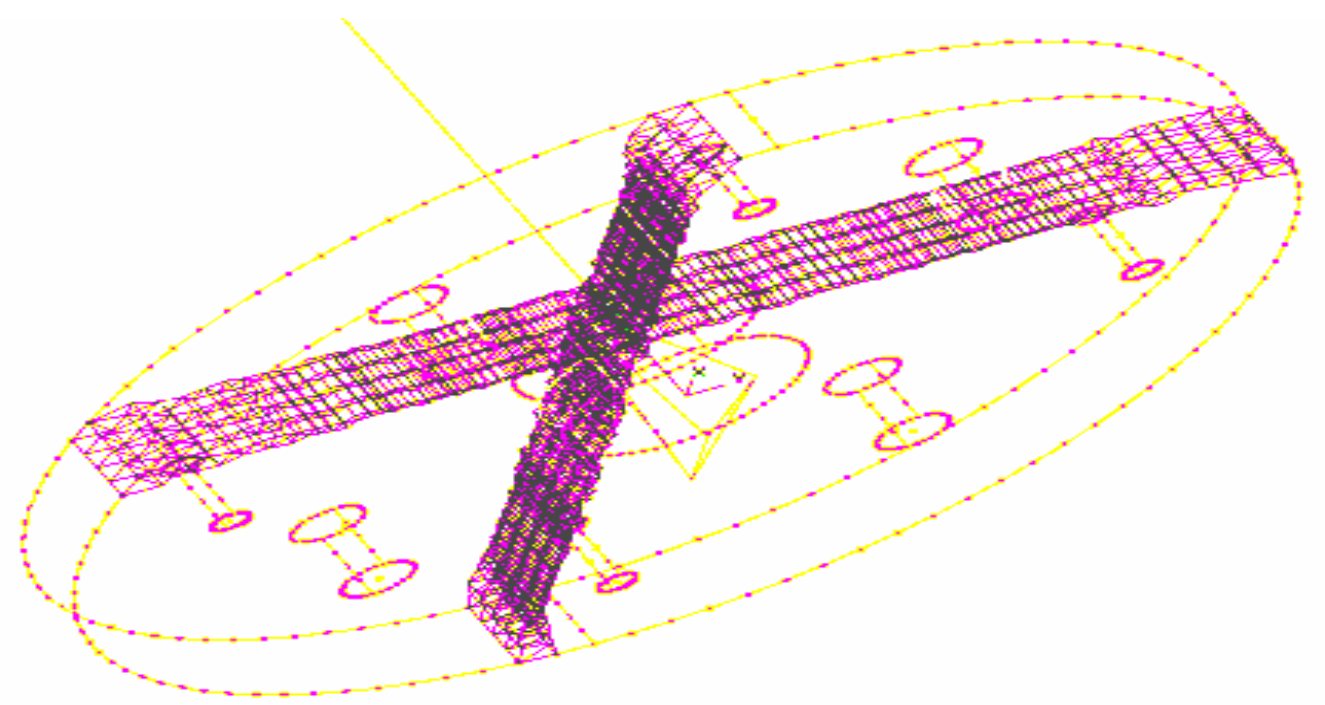

Figure 8: 3D CFDACE microphysiometer model showing the grid used

The complexity of the model was then increased to incorporate some of the more salient features of the experimental setup. To do this, two long pipes were added to the inlet and outlet of the previous model. As shown in Figure 9 the inlet and outlet 
boundary conditions were applied at the ends of these two long pipes. The basic meshing technique remains the same with the pipes extruded from surfaces meshes at the earlier inlet and outlet.

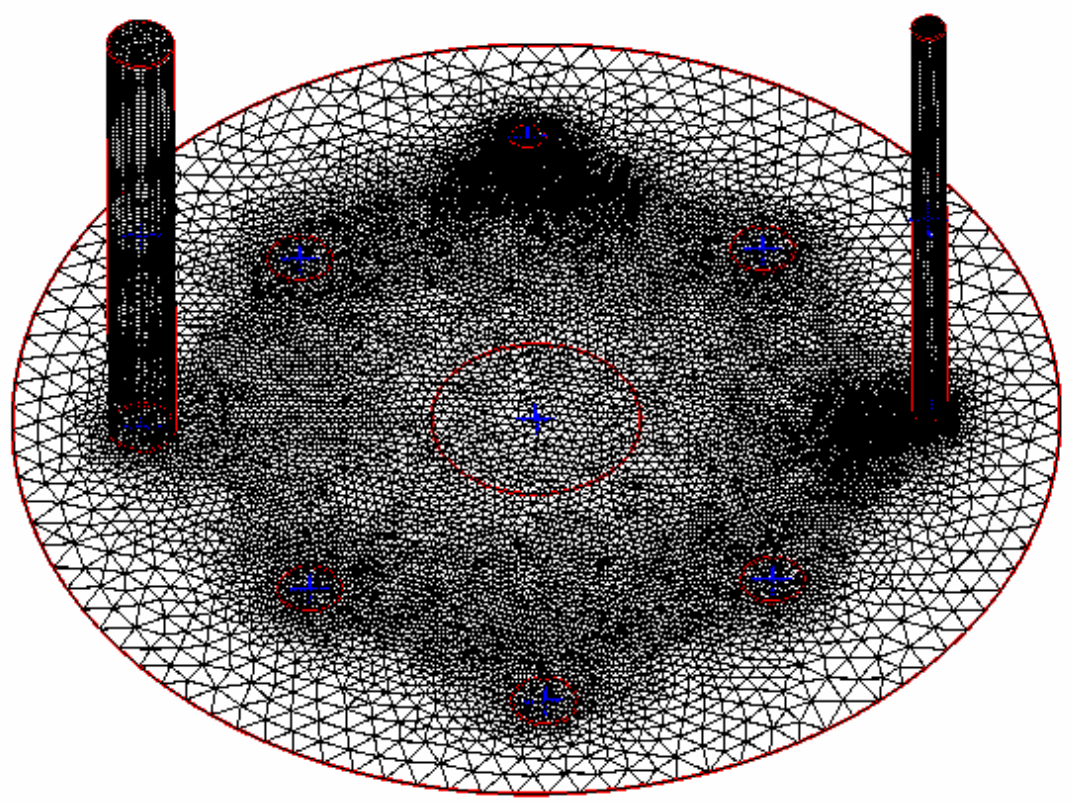

Figure 9: Meshed complex microphysiometer model

For detecting glucose the geometry has to be slightly modified to take into account the volume reaction taking place using Michaelis-Menten kinetics. The central sensor in the model was used to measure glucose. Hence an additional cylindrical volume has to be added just above the central sensor alone to create the volume required to allow the volume reaction to take place. The volume reaction boundary conditions were applied in this volume along with the rate reactions as described in section 2.4. Boundary conditions at all other surfaces are exactly the same as the earlier model and meshing was also done using the same techniques. A user-subroutine (Appendix A) was created to confine the enzyme reaction in the 
sensor region. More detailed studies will be done as soon as the constants that define the rate reaction factors are determined experimentally.

\subsection{Microphysiometer geometry for oxygen sensing with FLUENT}

For validation purposes, modeling was also done using Fluent. Figure 10 and 11 below shows the top view and side view of the model with the inlet, the outlet and sensor for the steady state solution. It also shows a few flow streamlines from inlet to outlet. The contours show the concentration level of oxygen in the microphysiometer chamber.
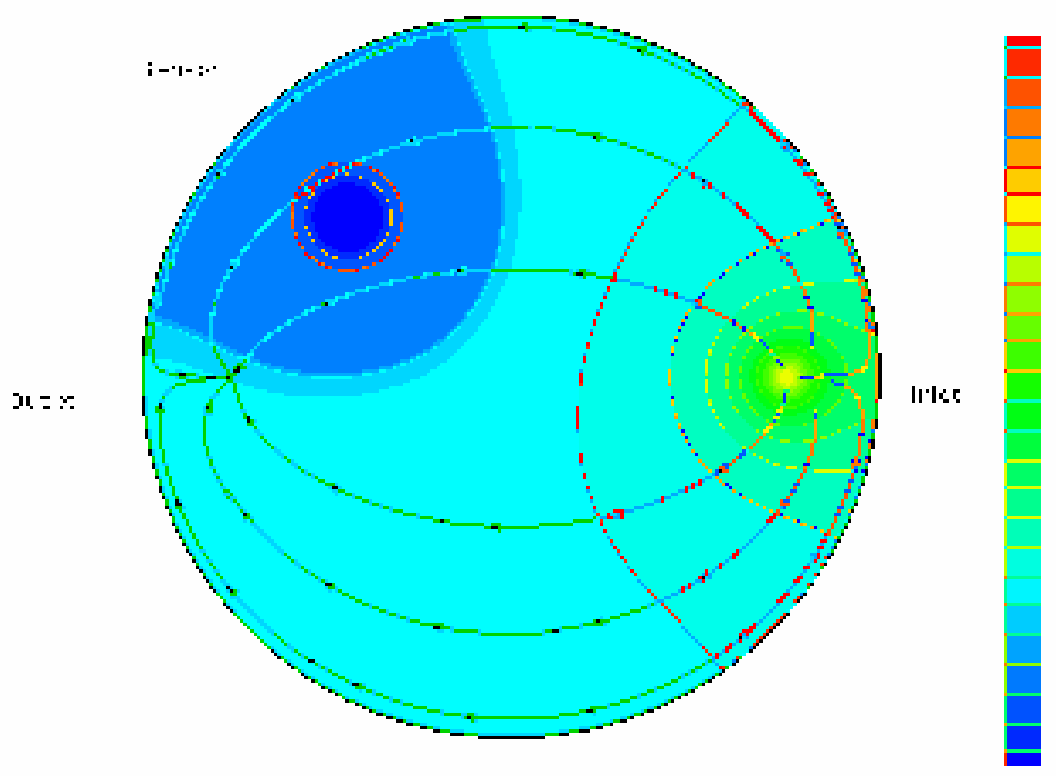

Figure 10: Top view of the 3D microphysiometer modeled using FLUENT. Streamlines and oxygen concentration profile are shown 


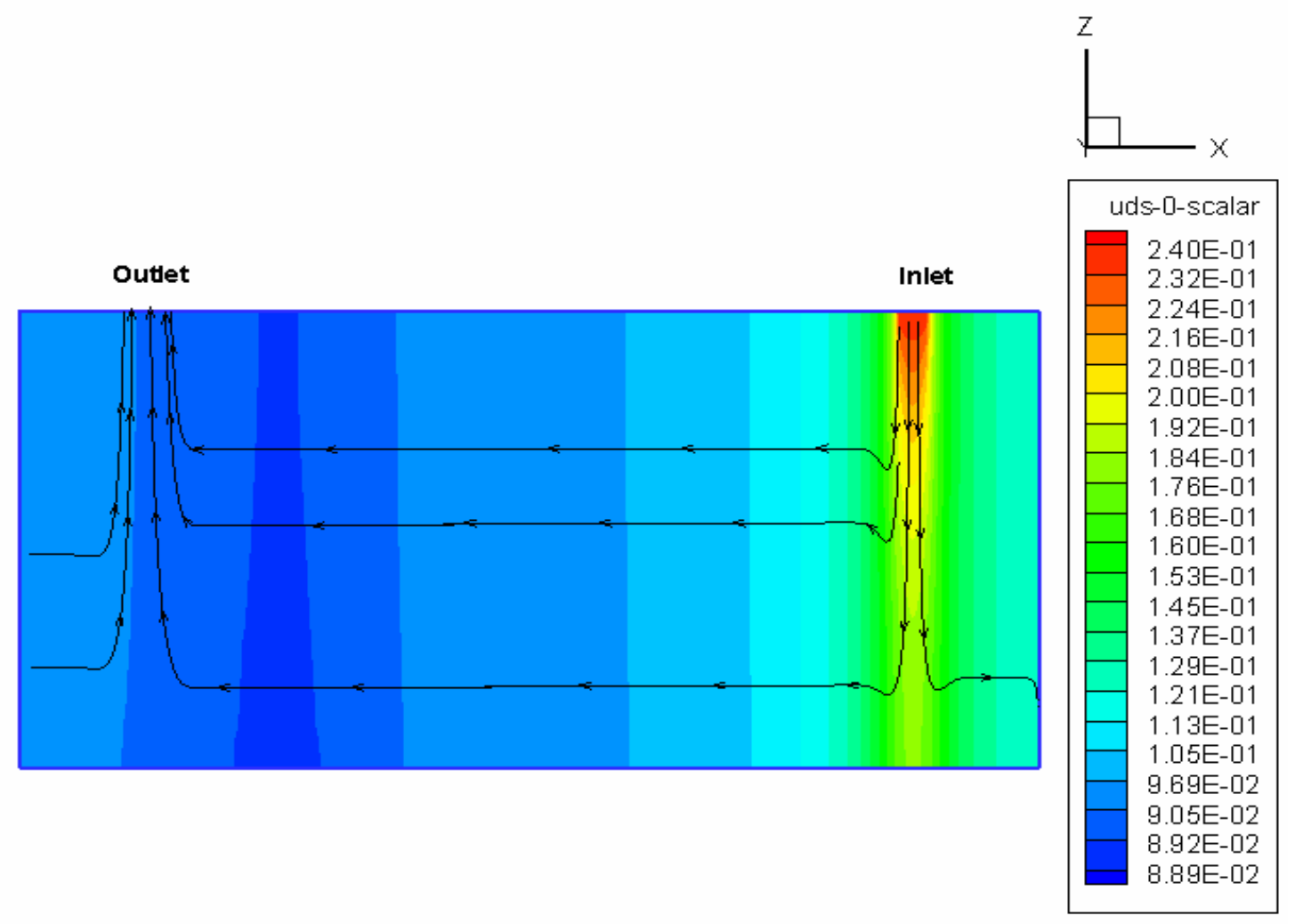

Figure 11: Side view of the 3D microphysiometer modeled using FLUENT showing streamlines and oxygen concentration profile.

\subsection{Grid convergence}

In order to maximize accuracy, one should use as many computational nodes as possible. Using two many nodes, however, is not practical as there is always only a finite amount of computational power. It is important to find a balance between the number of nodes and the accuracy of the solution. In this work the accuracy of the solution is determined by finding the root mean square concentration error. The dependence of the solution on the grid refinement is shown in figure 13. While using CFD-ACE it was found that for the model with 72,000 nodes, the ideal mix of accuracy and speed of convergence was achieved. To get this balance the first model 
was meshed coarsely and then the models were slowly modified by increasing the number of nodes in the geometry till the value of the concentration, stopped changing with $.0001 \mathrm{mM}$ tolerance. The Figures 12 and 13 give an idea of how the study was conducted.
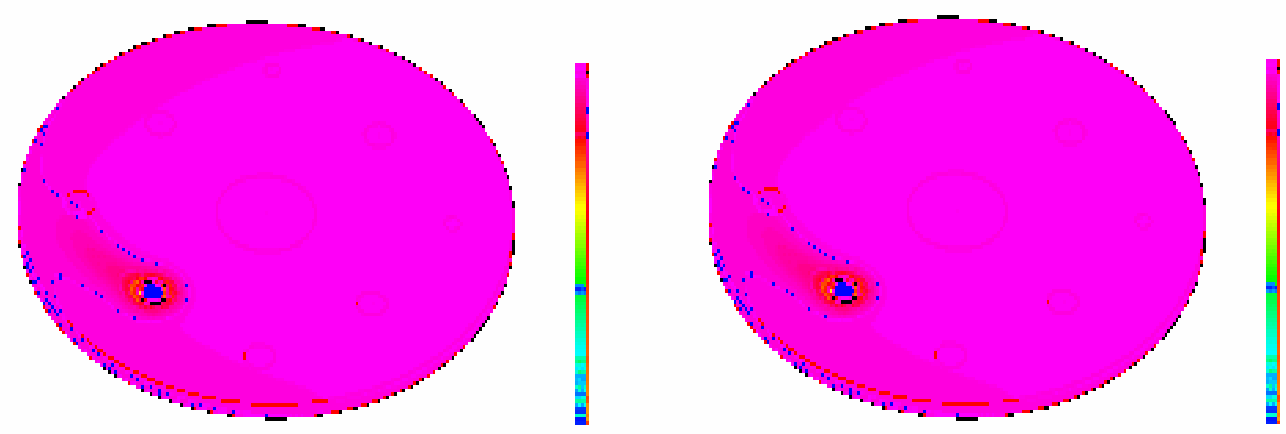

Figure 12: Results for CFDACE model with 72,000 and 150,000 nodes steady state solution with initial inlet concentration $=0.24 \mathrm{mM}$ and cell uptake rate $=-0.00025$ $\mathrm{mM} / \mathrm{secm} 2$

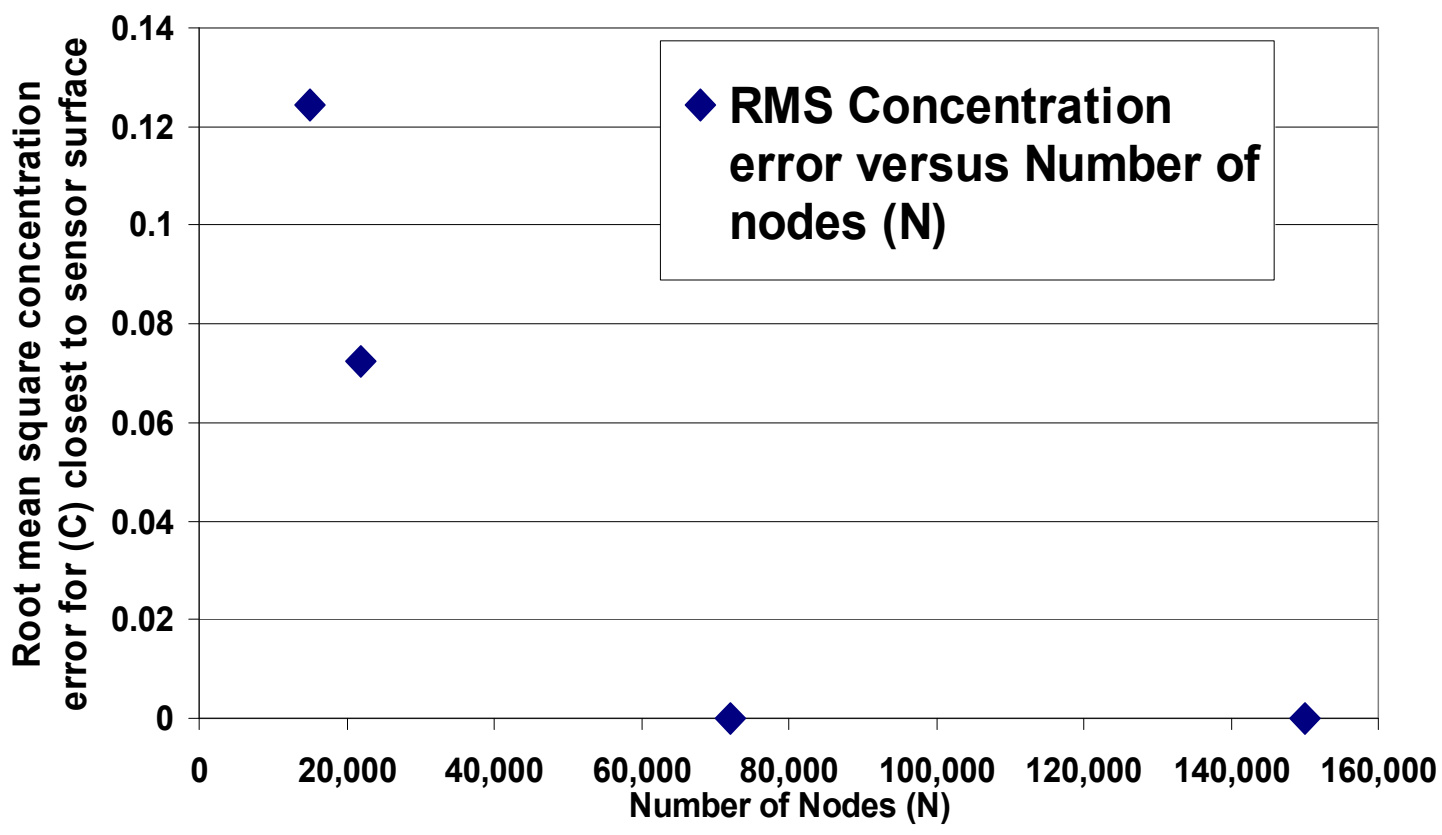

Figure 13: Comparison of different models with varying number of nodes to show grid convergence of the concentration error 
Figure 14 shows the grid convergence tests for the FLUENT model.
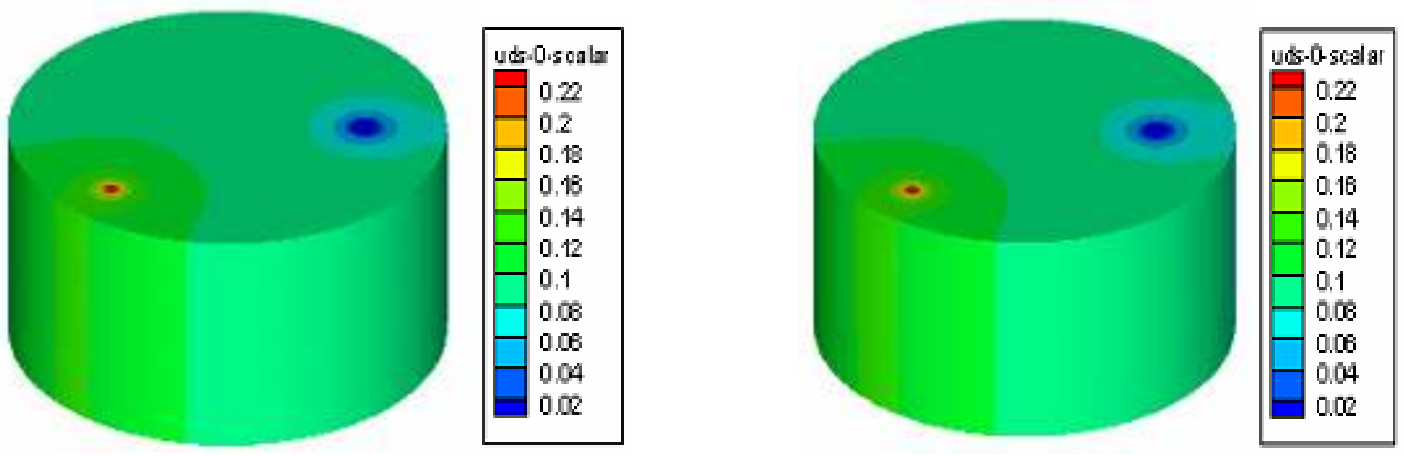

Figure 14: Results for FLUENT model with 500,000 nodes and 1,500,0000 nodes for steady state solution with initial concentration $=0.24 \mathrm{mM}$ and cell uptake rate $=-$ $0.00025 \mathrm{mM} / \mathrm{secm}^{2}$

Since both the models give essentially the same results the 500,000 model was used for further calculations. It was noticed that the FLUENT model ran significantly faster than the CFD-ACE model for this problem. The 500,000 node mesh in FLUENT took only a few hours more than the 72,000 node mesh in CFD-ACE. 


\section{CHAPTER IV}

\section{OVERVIEW OF RESULTS}

\subsection{Expectations}

The signal from the sensor can be expected to be different from the ideal response. When compared to the ideal signal the measured signal will have a phase lag as well as a reduction in magnitude. In the following sections, the results obtained with the computational model will be studied.

\subsection{Results with CFD-ACE model}

In this section, the microphysiometer with CFD-ACE. One of the outer sensors closest to the outlet is turned on, and the rest of the sensors are turned off. The sensor current is calculated from the local concentration gradient as shown in section 2.6. A first order and a second order method have been compared to calculate the concentration gradient. The first order method only takes one node closest to the sensor in the perpendicular direction, joins the concentrations at the wall and the node with a straight line, and calculates gradient from it. In this method the gradient remains the same at all points in between the two points. The second order method uses two nodes. It takes the concentration at two nodes and at the boundary and fits a curve using a Taylor series expansion. Here the concentration gradient changes as the distance from sensor changes. Here the perpendicular distance at which the concentration gradient is calculated is at zero distance from the sensor. 


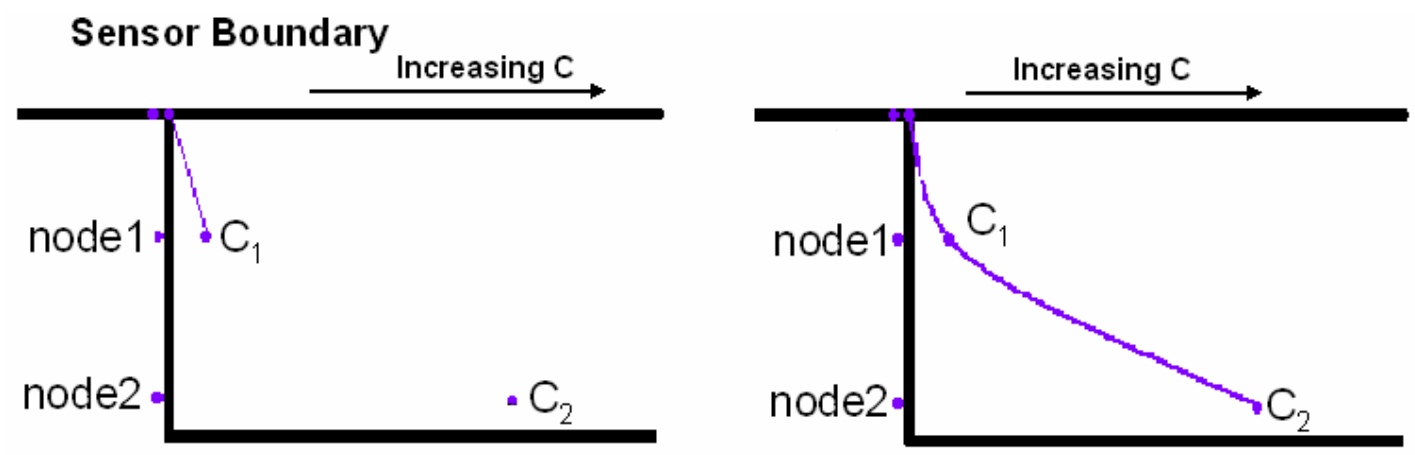

Figure 15: Shows first and second order gradient calculation methods

First the effect of varying the initial concentration $\mathrm{C}$ during the steady state solution is studied. Figures 16,17 and 18 compare the first order and second order methods for calculating the concentration gradient from which current can be computed. Figure 18 compares the first order approximation and the second order approximations at the same initial concentration. Figure 19 shows that there is almost a difference of up to $30 \%$ in the magnitudes of the two approximations. From the graphs it can be assumed that the second order calculations have better accuracy. So from hence forth, only second order calculations will be used 


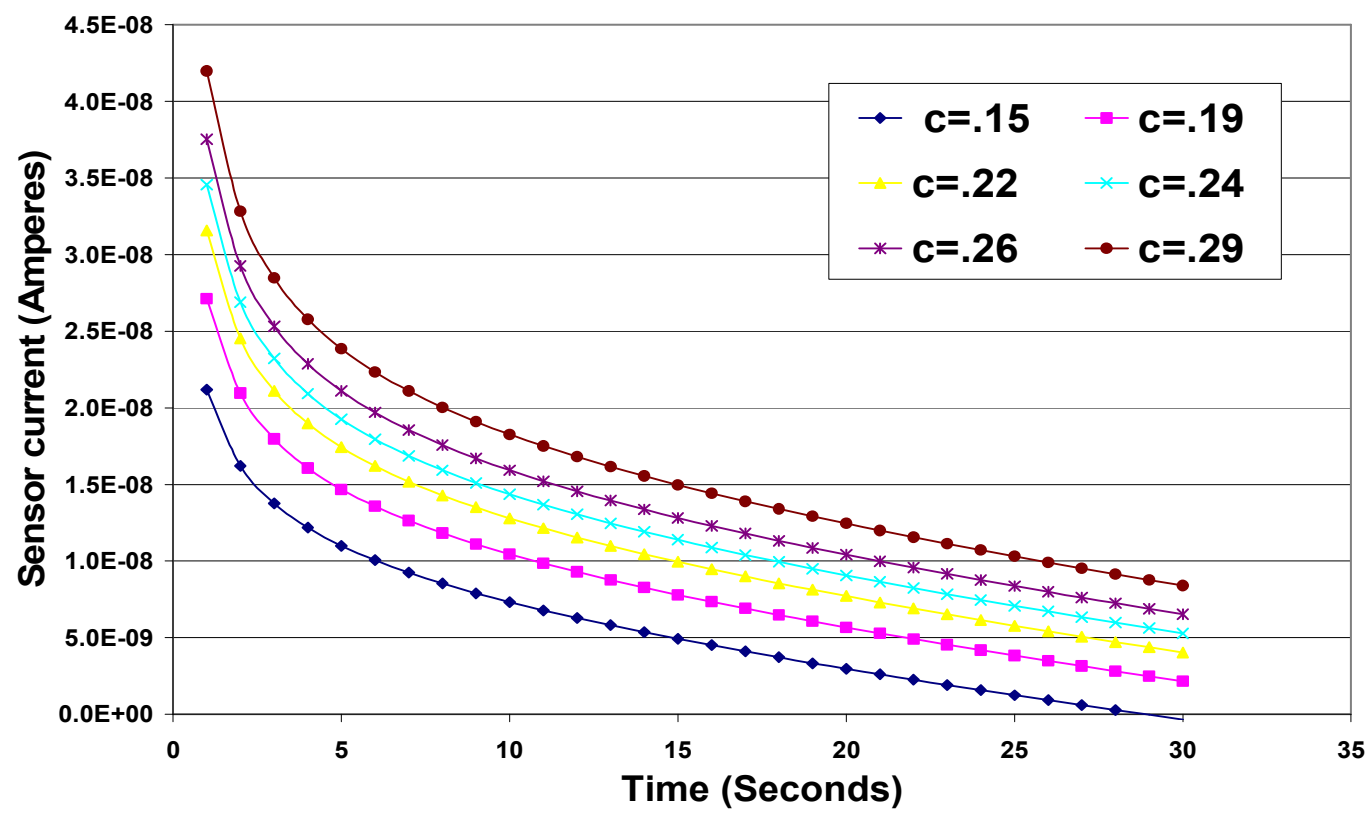

Figure 16: Dependence of the sensor response on the initial $\mathrm{O}_{2}$ concentration $\mathrm{C}$, given in $\mathrm{mM}$. Gradients are calculated using the first order approximation 


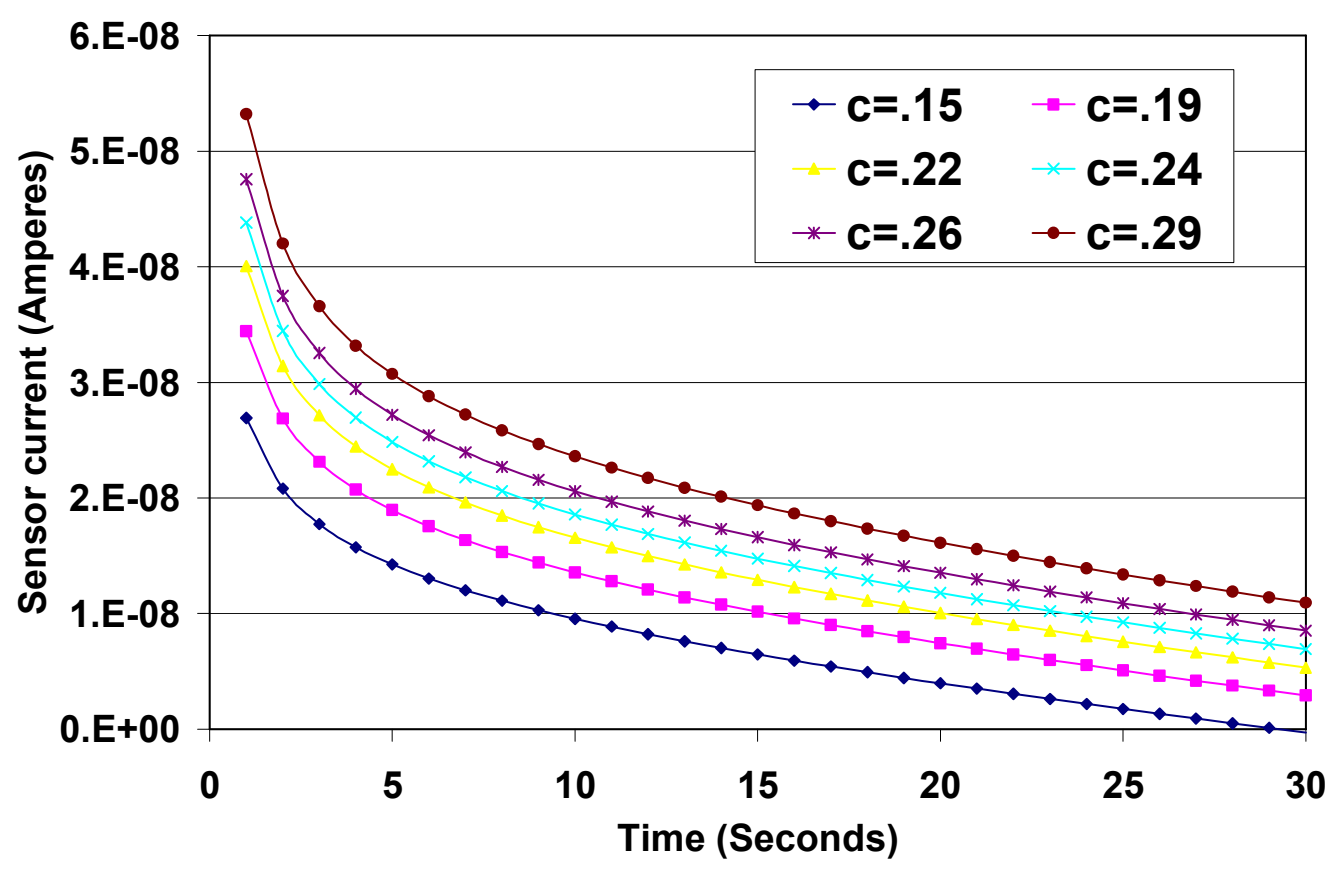

Figure 17: Dependence of the sensor response on the initial $\mathrm{O} 2$ concentration $\mathrm{C}$ in $\mathrm{mM}$. Gradients are calculated using the first-order approximation

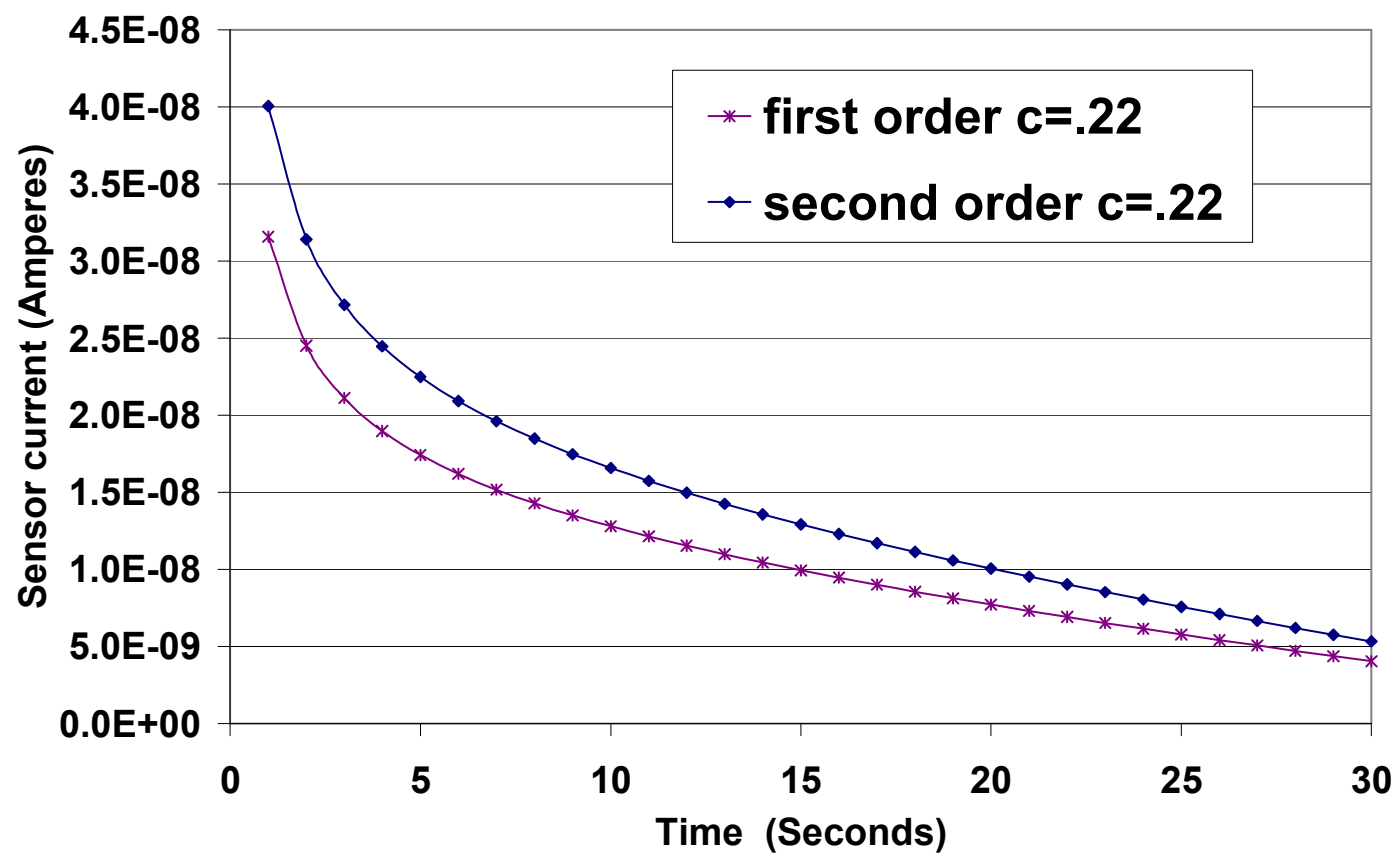

Figure 18: Comparison of first order and second order approximation of the gradient for an inlet concentration $\mathrm{C}=0.22 \mathrm{mM}$. 


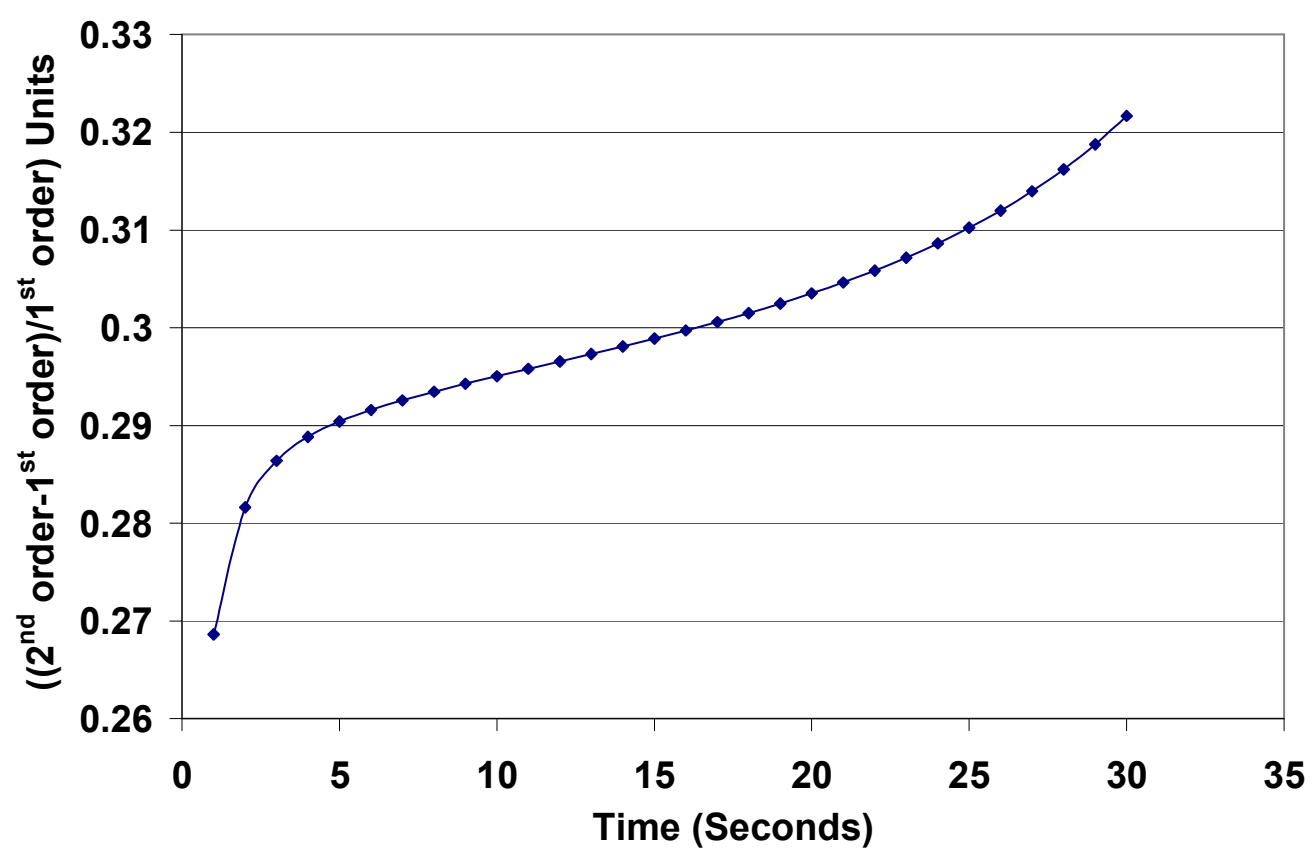

Figure 19: Relative difference between first order vs second order approximation for an inlet concentration $\mathrm{C}=.22 \mathrm{mM}$.

A parametric study to evaluate the effect of the cell flux consumption (qw) and how it affects the current output was done. Again first order and second order data were compared and it can be assumed that the second order is more accurate and will be used for further analysis (Figure 21). One parametric run is done with the cells totally killed or with the cell uptake flux set to zero to get the current output due to sensor alone. This data was then subtracted from the data where the cells have not been killed which allows us to steady the effect of the cells alone.

Total sensor output(Tso $)=$ Output from Sensor(So) + Output because of cells $(\mathbf{C o})$ 
The mathematical relation 4.1 shows how the total output is divided between the cells and the sensor. Figure 21 shows the comparison of the effect of both the cells and the sensor on the output current signal. It also looks at the difference between the first order and the second order results.

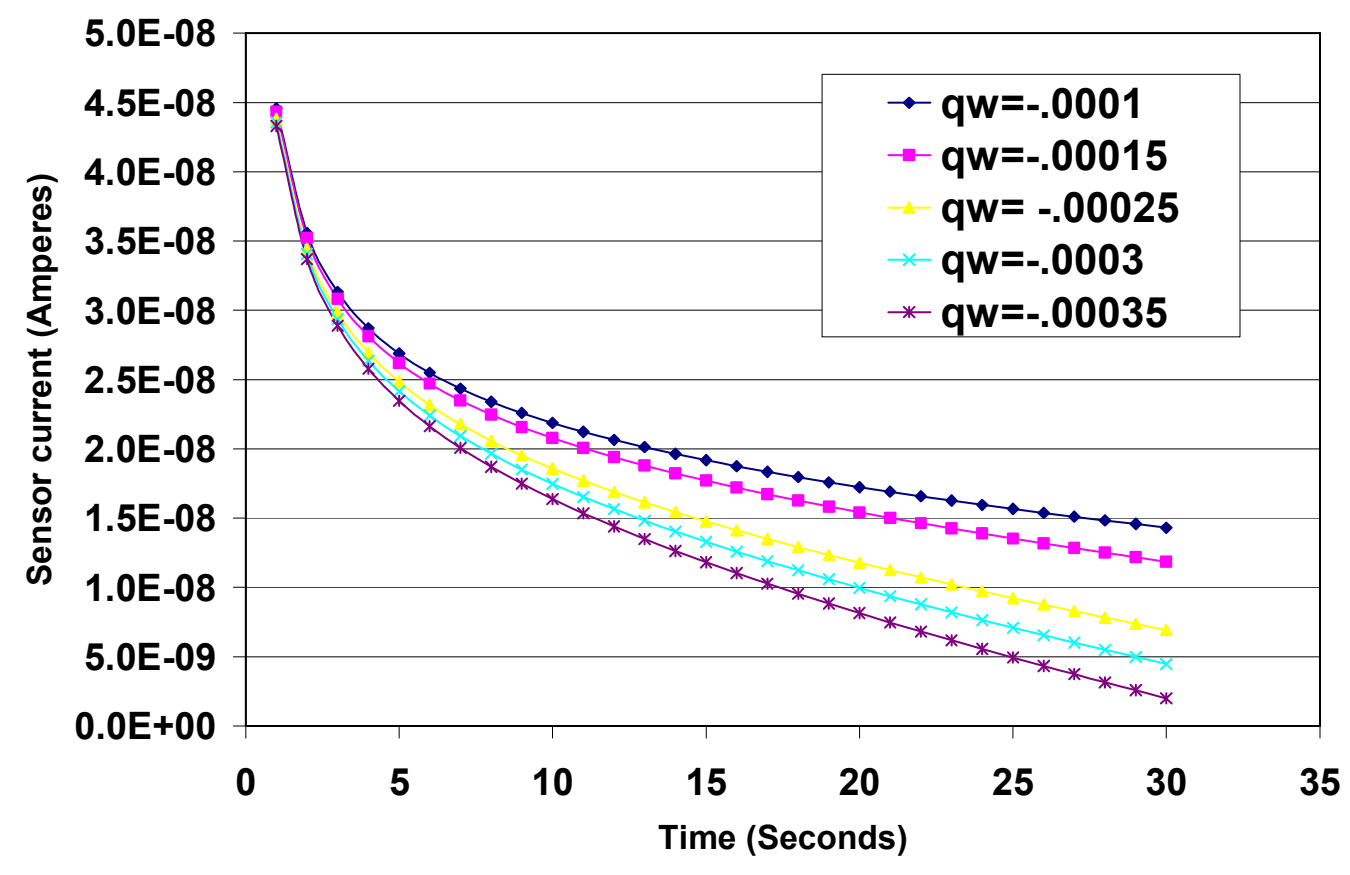

Figure 20: Parametric study of cell flux uptake (qw in $\mathrm{mM} / \mathrm{secm} 2)$. Tso output of sensor for Second order and $\mathrm{C}=.24 \mathrm{mM}$ 


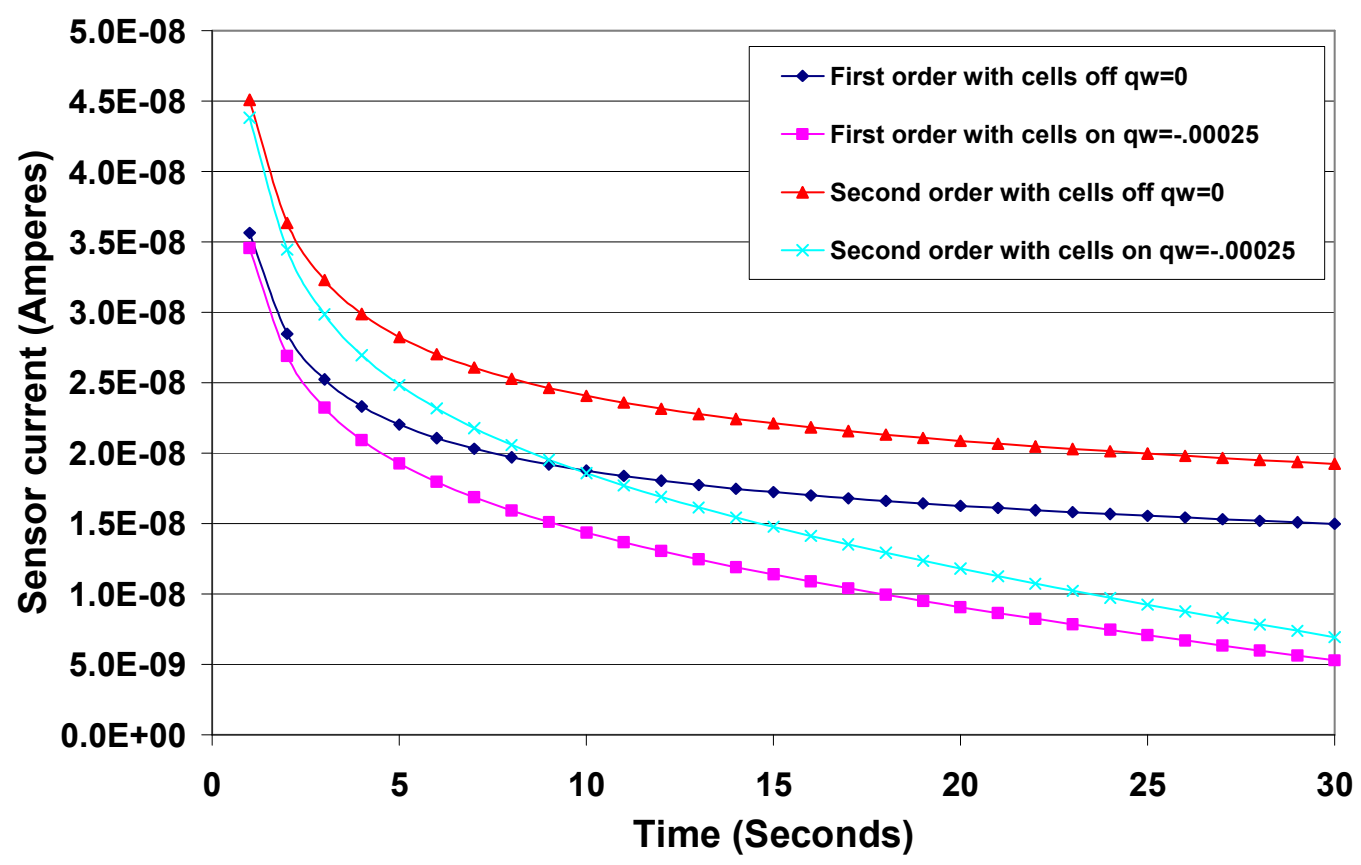

Figure 21: Comparing Tso and Co for $\mathrm{qw}=-.00025 \mathrm{mM} / \mathrm{secm}^{2} \& \mathrm{C}=.24 \mathrm{mM}$. Concentration gradients calculated using first and second order methods.

Both the sensor and cells consume oxygen in the microphysiometer chamber.To isolate the effect of the cells alone on the output, the data measured with cells uptake rate switched off, is subtracted from the normal data sets. This gives an almost linear set of graphs as shown in Figure 22. 


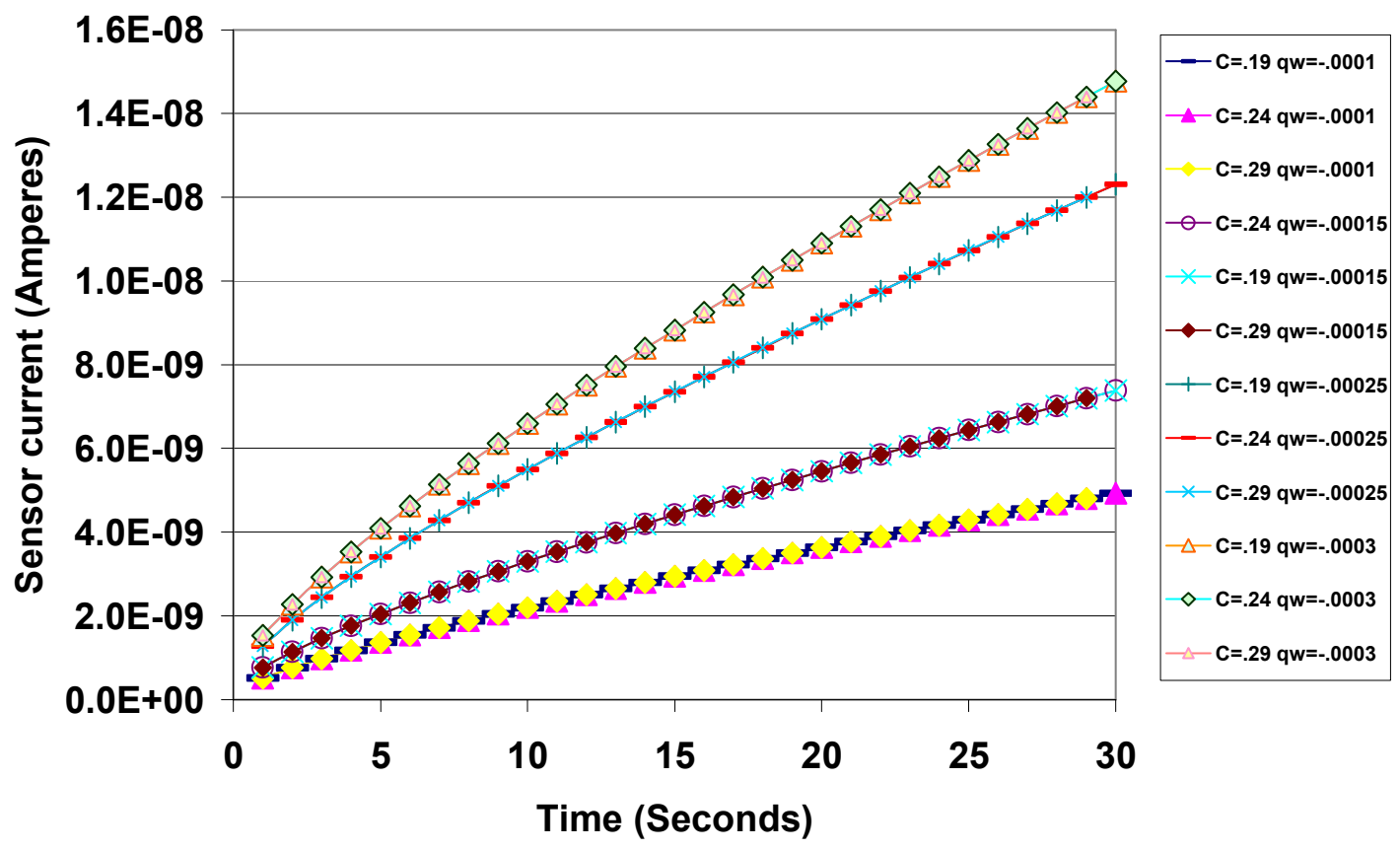

Figure 22: The Co output of sensor at different inlet concentration (C in $\mathrm{mM})$ and cell uptake rates (qw in $\mathrm{mM} / \mathrm{secm}^{2}$ ).

Figure 22 shows that once the effect of the sensor on the output is removed, the initial inlet concentration does not affect the output of the sensor due to cells alone. All the plotted data for various initial concentrations (C) having the same cell flux uptake (qw) fall on top of each other. This is an important observation because it is physically difficult for the experimental group to determine the inlet concentration accurately. The modeling shows it is not required to have a very high accuracy of the inlet concentration provided the effect of the cell uptake rate is looked at independently without the effect of the sensor. The important thing in the study is that for each data cycle a separate cell's off data run should also be conducted and then be subtracted from the original data to get the effect of the cells alone. Only then does the data fall on top of each other. This happens because any effect the initial 
concentration (C) has on the data is also present in the cells off data ( $\mathrm{qw}=0$ $\mathrm{mM} / \mathrm{secm}^{2}$ ) which gets cancelled on subtraction and hence the plots fall on top of each other.

To show that the effect of varying the inlet concentration does not affect the output generated by cells alone, the time factor was taken out of the comparison. Figure 23 shows the time averaged comparison of data taken from the previous graph versus cell uptake rate for various inlet concentrations. It can be seen that all the linear graphs fit onto one straight line further proving that the inlet concentration does not affect the current values if cells off current signal is taken into account.

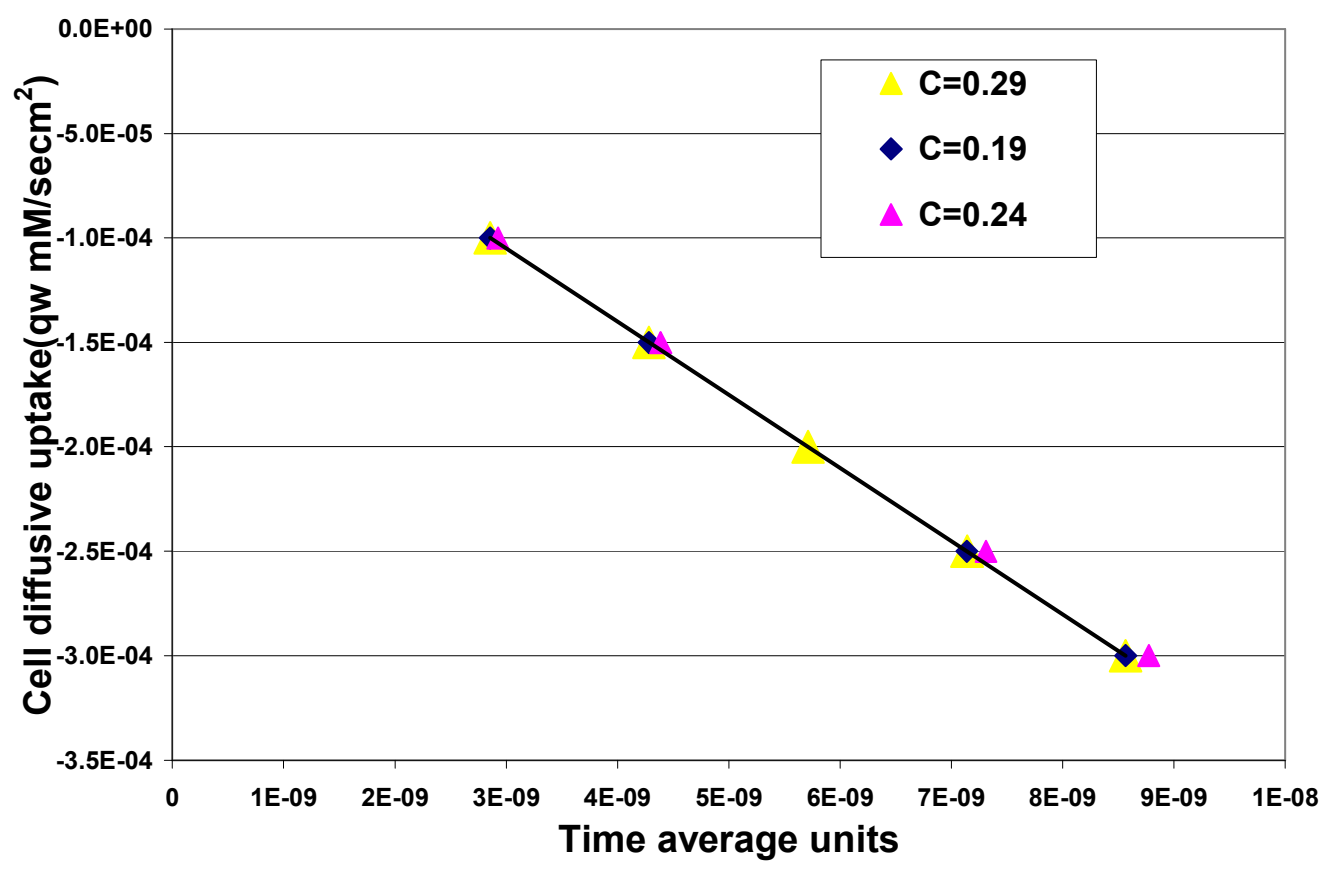

Figure 23: Time averaged versus cell uptake rates $\left(\mathrm{qw}\right.$ in $\left.\mathrm{mM} / \mathrm{secm}^{2}\right)$ for various inlet concentrations $(\mathrm{C}$ in $\mathrm{mM})$ 


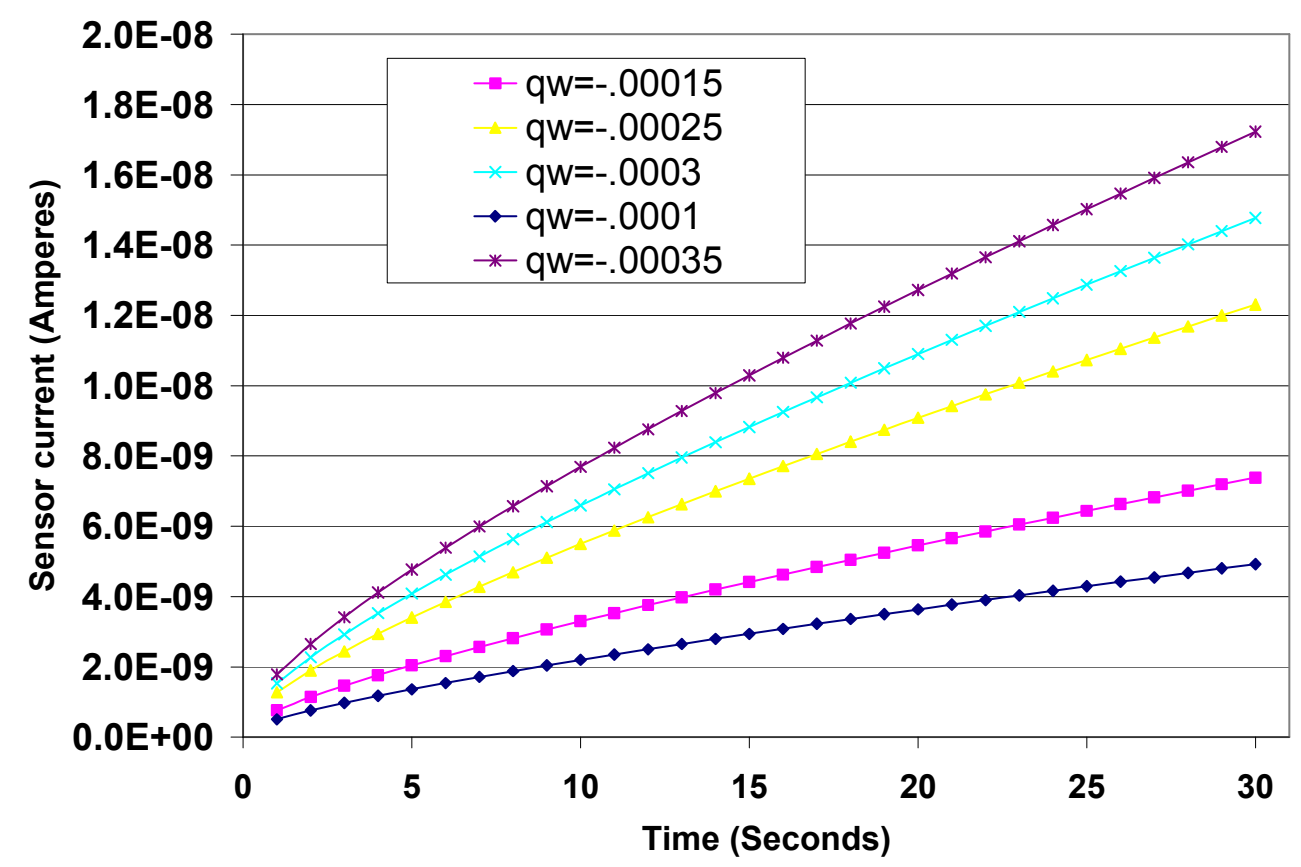

Figure 24: Co sensor outputs for different Cell uptakes ( $\mathrm{qw}$ in $\mathrm{mM} / \mathrm{secm}^{2}$ ) and initial concentration $\mathrm{C}=.24 \mathrm{mM}$.

In Figure 24 we see that varying the cells uptake (qw) does affect the current output of the sensor even when only the effect of the cells is taken into account.

In order to determine if a mathematical model could be found to predict the effect of varying cell uptake (qw), a curve fit of $3^{\text {rd }}$ order polynomial of the form $\left(a x^{3}+b x^{2}+c x+d=0\right)$ was done for each of the data sets. In figure $25,26,27$ and 28 another curve fit is used to see the trends in the coefficients of the $3^{\text {rd }}$ order curve fit. It was found that all the coefficients of the $3^{\text {rd }}$ order polynomial curve fit have a linear trend as shown in the graphs. The trend line equations are also displayed in the graph. 


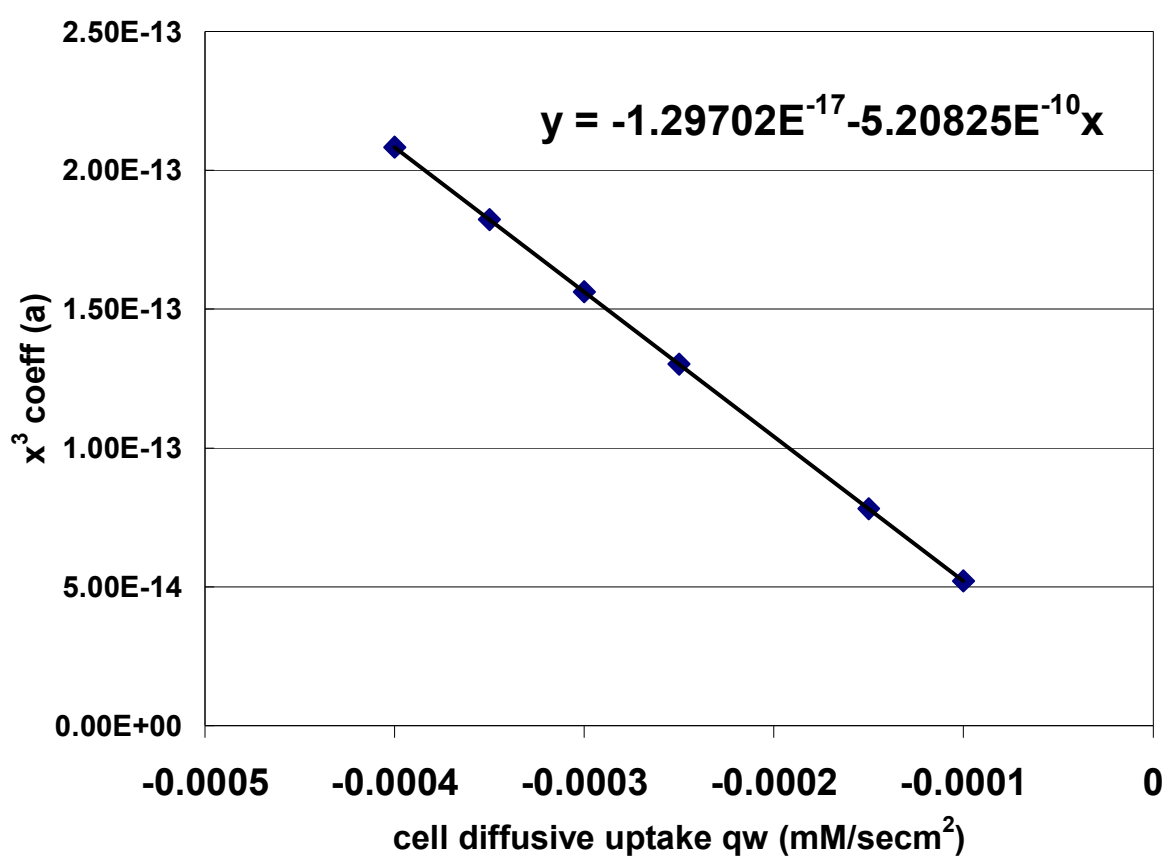

Figure 25: Dependents of coefficients of $x^{3}$ (i.e. a) on the cell uptake rate

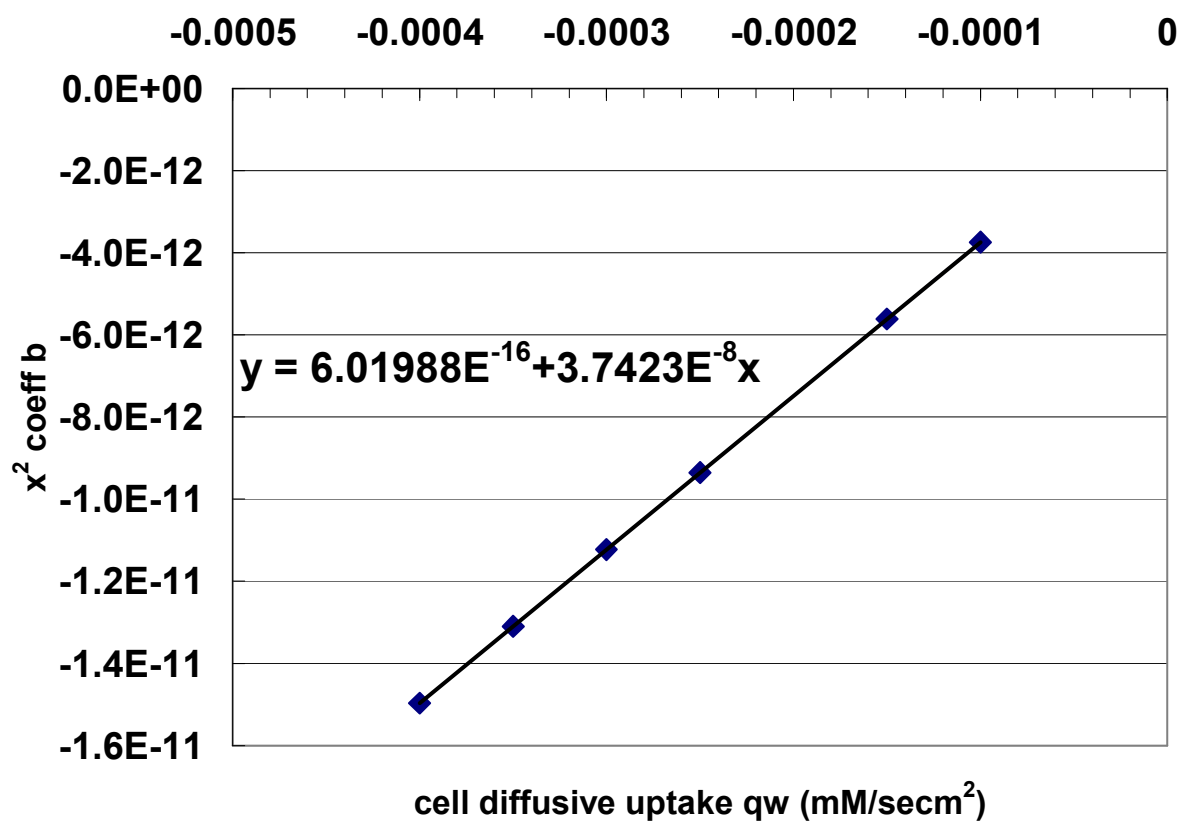

Figure 26: Dependents of coefficients of $x^{2}$ (i.e. b) on cell uptake rate 


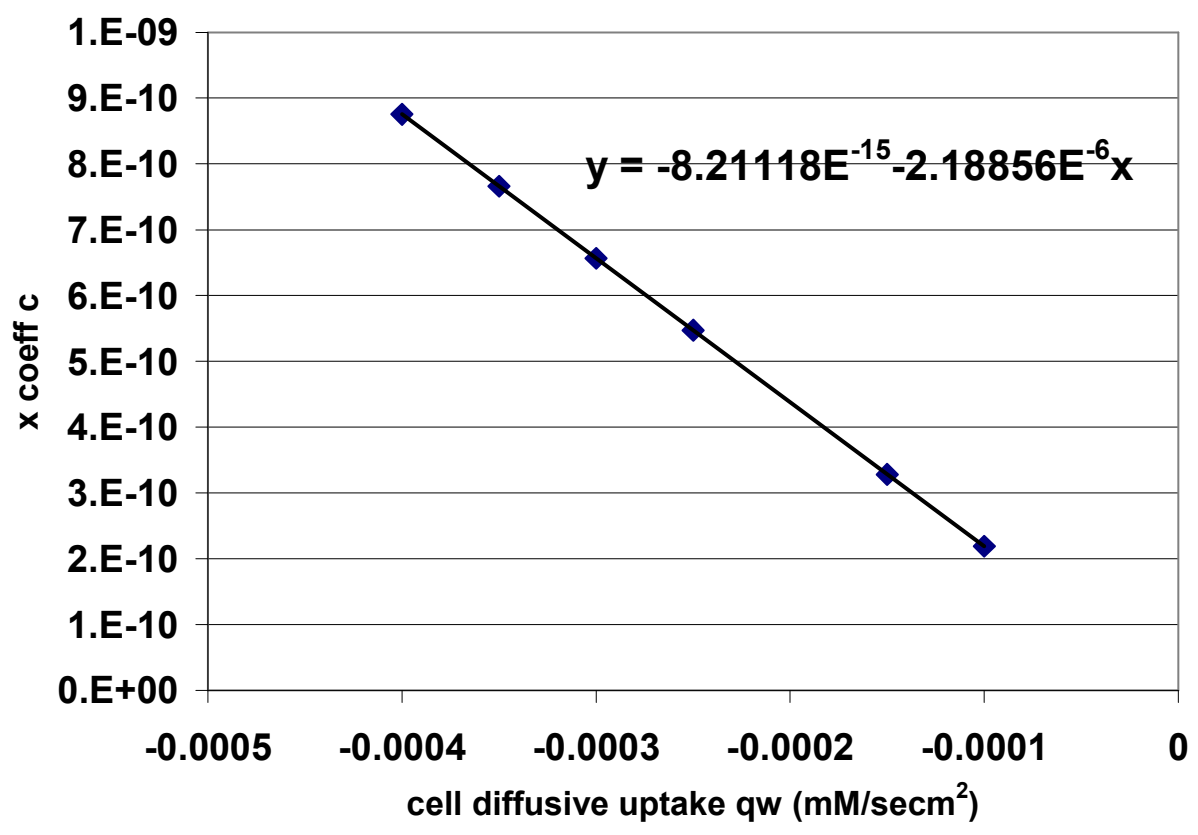

Figure 27: Dependence of coefficients of $x$ (i.e. c) on the cell uptake rate

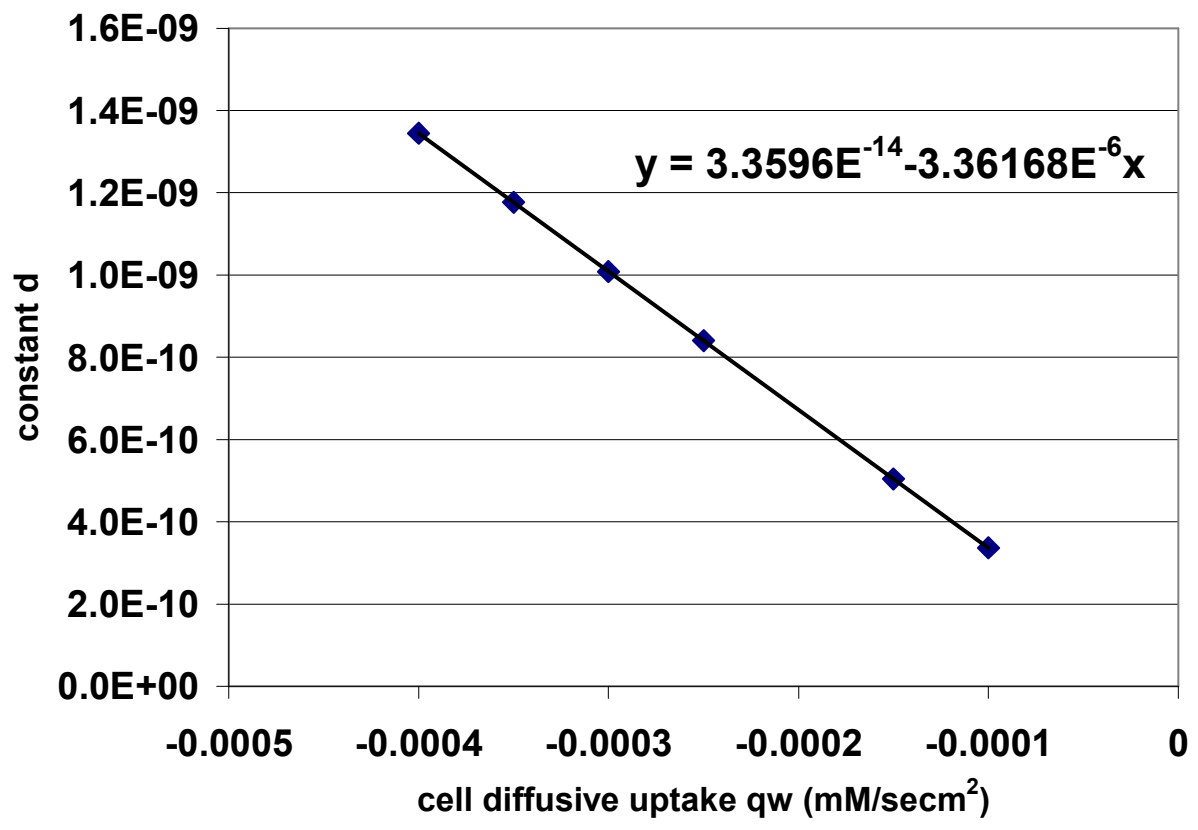

Figure 28: Dependence of constant coefficients (i.e. d) on the cell uptake rate 
Using the results from figure $24,25,26$ and 27 , it is was found possible to accurately predict the response of the computational model using

$$
\begin{aligned}
\text { Current } & =\left(3.3596 \mathrm{E}^{-14}-3.36168 \mathrm{E}^{-6} \times(\mathrm{qw})\right)+ \\
& \left(-8.2118 \mathrm{E}^{-15}-2.18856 \mathrm{E}^{-6} \times(\mathrm{qw})\right) \times(\text { Time }) \\
& +\left(6.01988 \mathrm{E}^{-16}+3.742 \mathrm{E}^{-8} \times(\mathrm{qw})\right) \times(\text { Time })^{2} \\
& +\left(-1.29702 \mathrm{E}^{-17}-5.20825 \mathrm{E}^{-10} \times(\mathrm{qw})\right) \times(\text { Time })^{3}
\end{aligned}
$$

where qw is the cell uptake $\left(\mathrm{mM} / \mathrm{secm}^{2}\right)$ and Time is in seconds.

The process of finding the cell uptake for any given set of curves has also been automated. First a $3^{\text {rd }}$ order polynomial is found to fit the data. Then from the earlier set of linear coefficient graphs four values of cell uptakes can be got that lie very close to each other. Now the least squares method is used to match the data to the exact cell uptake using the modeling equation given earlier.

\subsection{Modeling with FLUENT (Preliminary studies)}

An alternative model was also created using FLUENT. As of now only steady state modeling has been completed using FLUENT. Figure 28 shows a parametric preliminary study undertaken to find the range of qw for which the steady state solution still has positive concentration in the microphysiometer chamber 


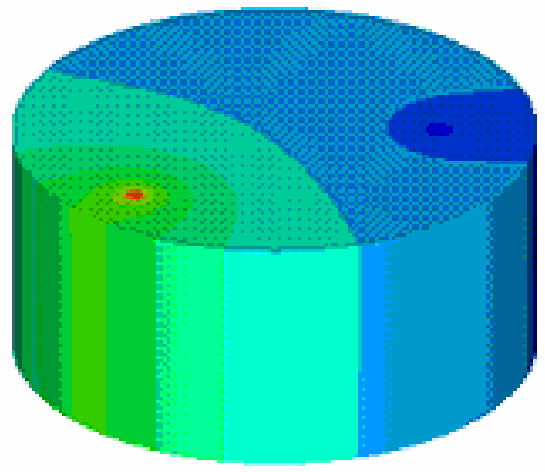

A.

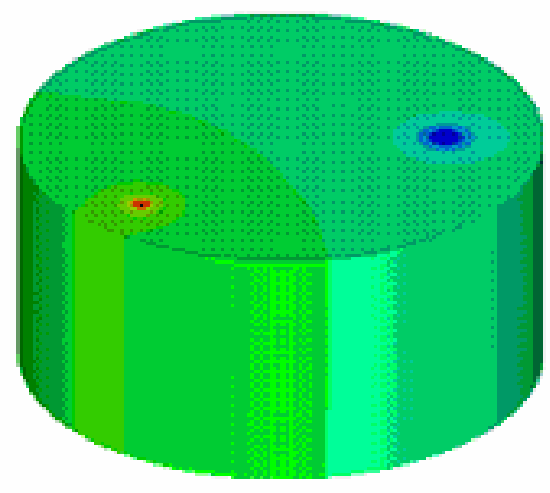

C.

Figure 29: Shows 3D model microphysiometer data modeled in FLUENT for steady state solutions with varying qw. A) $\left.\mathrm{qw}=2.5 \mathrm{E}-5 \mathrm{mM} / \mathrm{secm}^{2} \mathrm{~B}\right) \mathrm{qw}=1.5 \mathrm{E}-5 \mathrm{mM} / \mathrm{secm}^{2}$ C) $\left.\mathrm{qw}=1 \mathrm{E}-5 \mathrm{mM} / \mathrm{secm}^{2} \mathrm{D}\right) \mathrm{qw}=2 \mathrm{E}-5 \mathrm{mM} / \mathrm{secm}^{2}$

From figure 28 it is possible to determine that any cell intake above the cell flux of $\mathrm{qw}=2.5 \mathrm{E}^{-5} \mathrm{mM} / \mathrm{secm}^{2}$ results in negative values in the microphysiometer chamber for the steady state solution. Thus for further transient state studies the limiting range of the cell flux uptake of the cell is between $1 \mathrm{E}^{-5} \mathrm{mM} / \mathrm{secm}^{2}$ and $2 \mathrm{E}^{-5}$ $\mathrm{mM} / \mathrm{secm}^{2}$. 


\subsection{D model comparisons with experimental data}

In this section the modeled sensor response is compared with the experimental data obtained from the microphysiometer by the VIIBRE experimental group. (Eklund et al. 2004).

The modeled sensor measurements used for the comparison include both the effect of the cells and the sensor, because in the actual experiments the output current from the sensor includes both these effects. In order to compare the two sets of data it is necessary to match the initial points of the two data sets.

Figure 30 compares the modeled data and the experimental data for one oxygen sensor. The initial data for the transient solution is applied at 51 seconds. The modeled data has been scaled to match the experimental data. The comparison also takes into account the base current output (27nA) present in the experimental setup even when the sensor is not on. This base current is assumed to be $30 \%$ of the current measured by the experiments with the microphysiometer. 


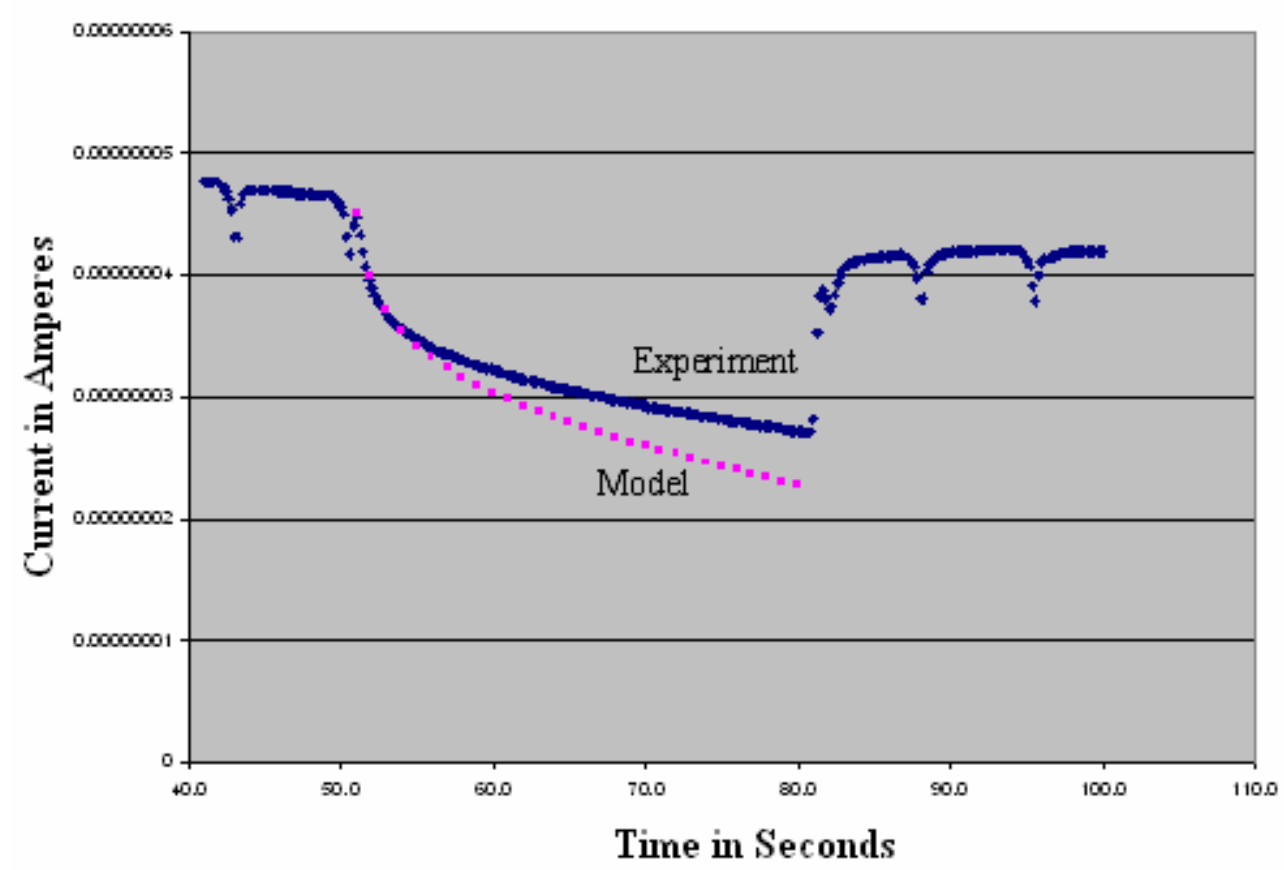

Figure 30: Comparing experimental and Modeled data for $\mathrm{C}=0.24 \mathrm{mM}$ and $\mathrm{qw}=-$ $0.00025 \mathrm{mM} / \mathrm{secm}^{2}$

A parametric study was performed in order to study the effects of various base currents and comparisons were made with two experimental sets of data (Figure 31). The first set of data was taken at the beginning of the experimental run and the second set of data was taken at the end of the same experimental run. The initial model data was matched to these two experimental data sets at the point when the flow stopped. Towards the end of the experimental cycle there is a gradual decrease in the strength of the signal that affects matching. 

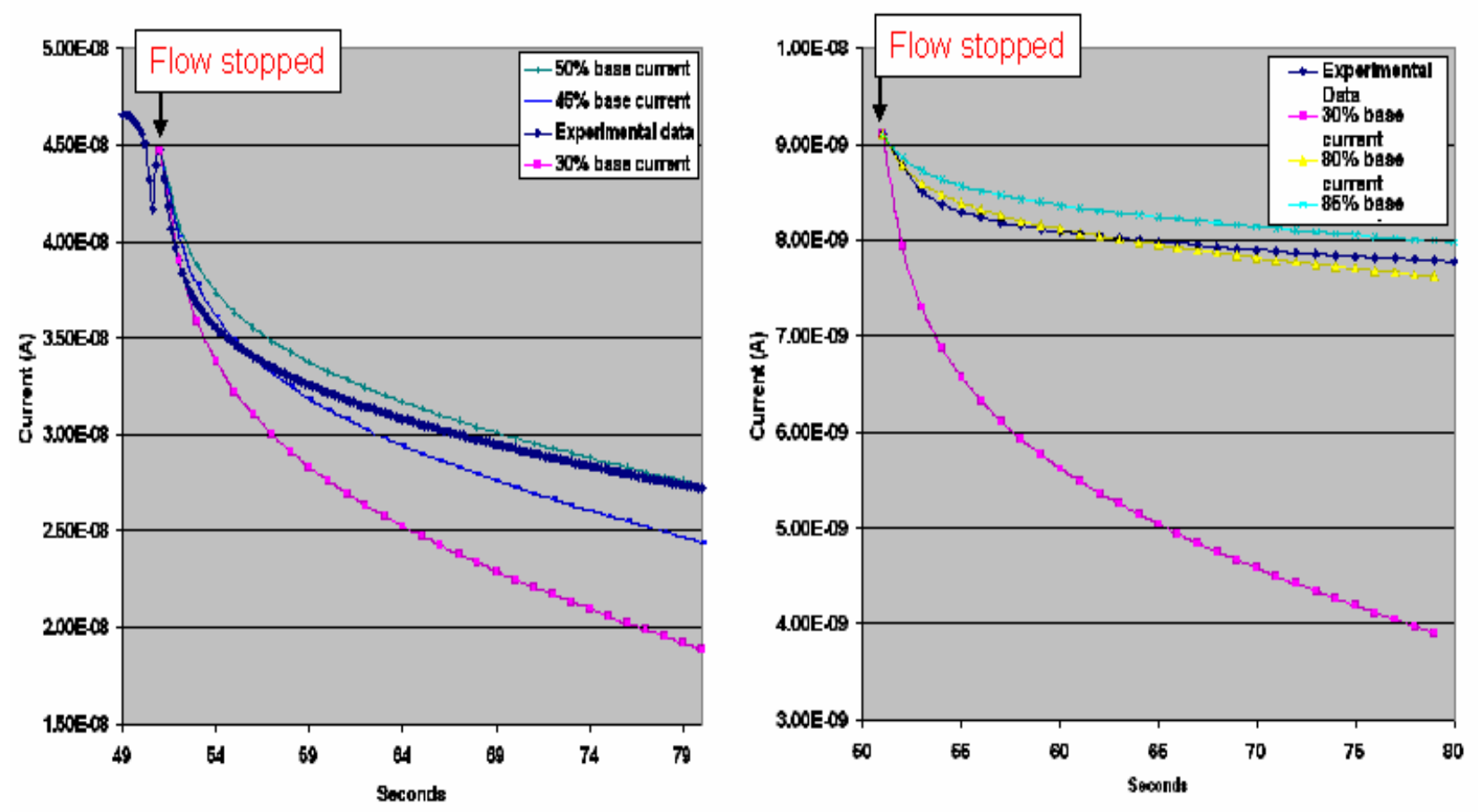

Figure 31: Comparing modeled data with different base currents for two different sets of data $\mathrm{C}=0.24 \mathrm{mM} \& \mathrm{qw}=-0.00025 \mathrm{mM} / \mathrm{secm}^{2}$.

From figure 31 it is seen that, the magnitudes of the model results do not match very well. If the initial points are scaled, the final points do not match and if the final points are scaled and matched then the initial points do not match. Thus there are still some geometric and physical characteristics in the experimental procedure that are not being depicted in the current model. On closer scrutiny of the experimental setup, it was found that the valves closing the inlet were at the end of long tubes leading into the cell chamber, and it takes this valve a couple of seconds to totally shut off the flow. A long pipe segment was thus added to both the inlet and the outlet of the microphysiometer model (section 3.3). Also the inlet boundary condition was assigned an exponentially decreasing function to closely depict the time it takes for the valve to actually shut down the flow. Both of these physical aspects were 
incorporated into a new redesigned geometric model. The updated model and graphs of current versus time are shown in figure 32.

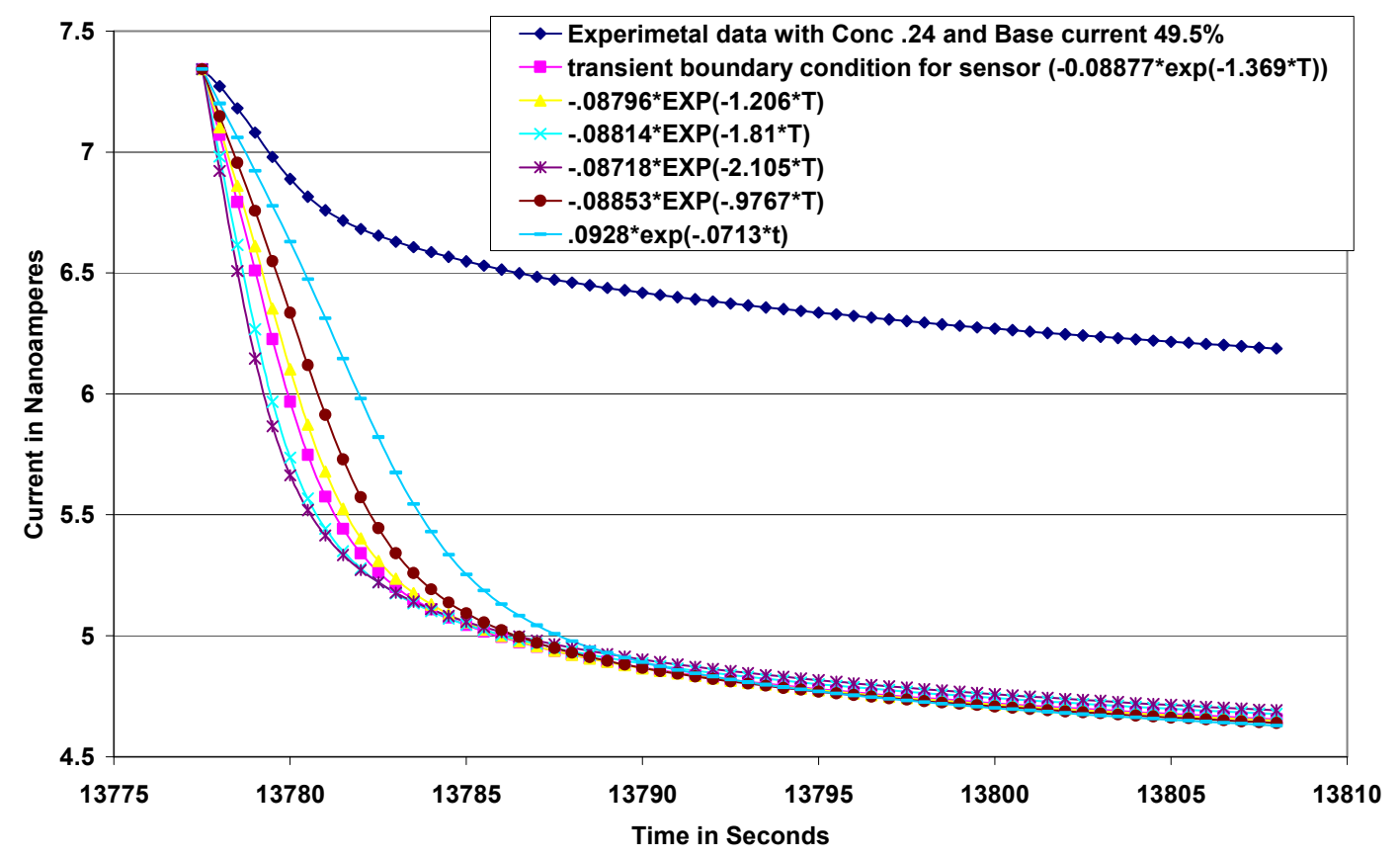

Figure 32: Comparison of experimental data and modeled data for initial concentration $\mathrm{C}=0.24 \mathrm{mM}$ at base current $49.5 \%$ base current with different exponential inlet boundary conditions

It can be seen that at the initial stages of the transient step the shape of the modeled data is similar to the experimental data, but there is still a small difference in the magnitude even after applying exponential inlet boundary conditions to the steady state solution (the initial condition for the transient state).

It is difficult for the experimental VIIBRE group to accurately measure the inlet concentration (C), which motivated the parametric study to examine the effect of initial concentration of $\mathrm{O}_{2}$. A comparison of these computational results with the experiments is shown in Figure 33. 


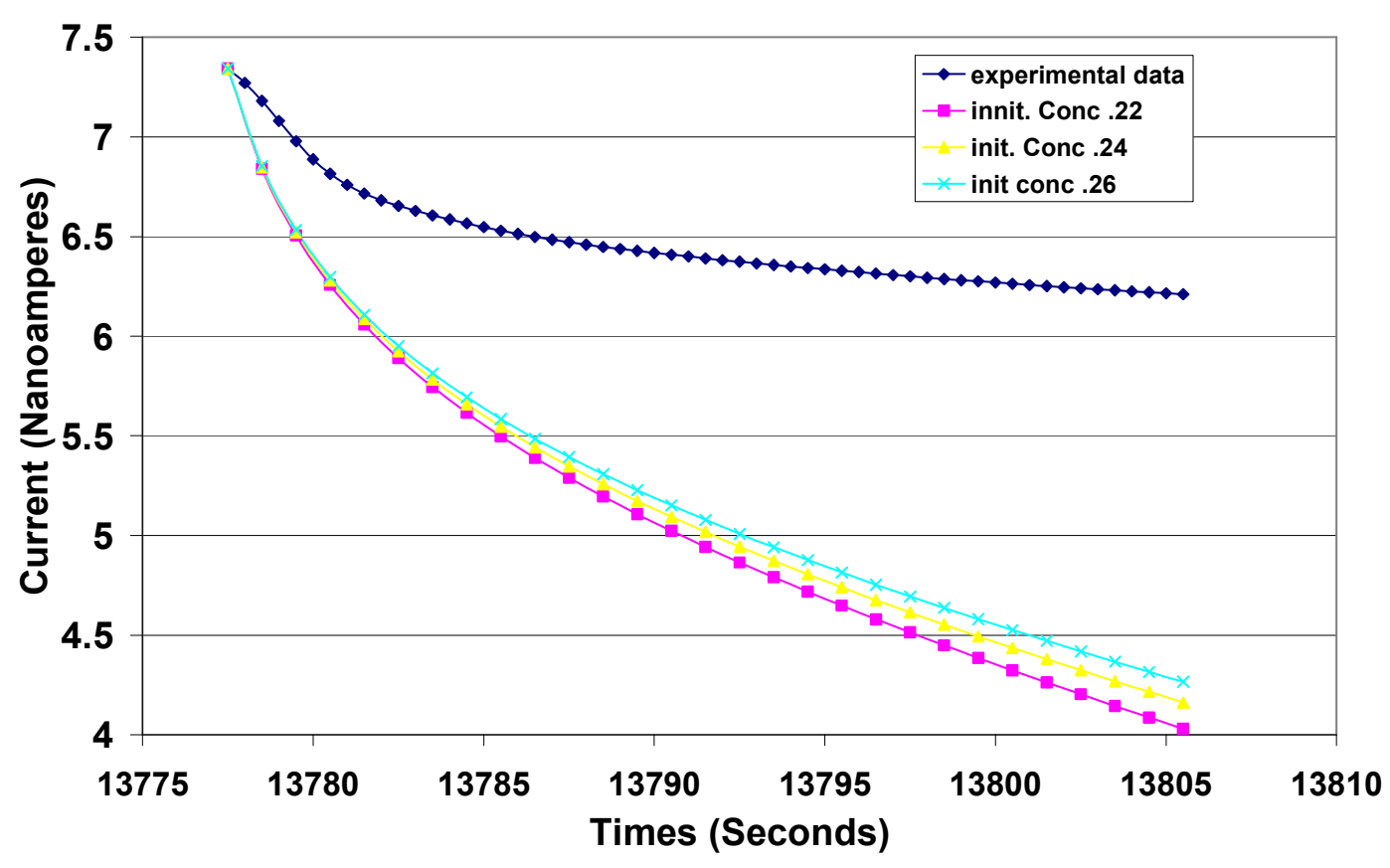

Figure 33: Shows the comparison between experimental and modeled data for varying initial concentrations $(\mathrm{C}$ in $\mathrm{mM})$

A change in the inlet concentration affects the total output current, and this effect increases as time increases, but a huge difference still remains between the computational results and the experimental data. A least squares comparison was performed to determine the base current for which the last 10 seconds of data best matches the experimental current. This base current of was found to be $49.5 \%$ of the total sensor current.

Another comparison was done by varying the cell flux boundary condition at the cell wall. This also gives similar results obtained by varying the initial conditions except that here the change is more pronounced in magnitude (Figure 34). It can be 
seen that the cell flux boundary condition has a much greater impact on the sensor output, and the magnitude of this effect also increases as time goes on.

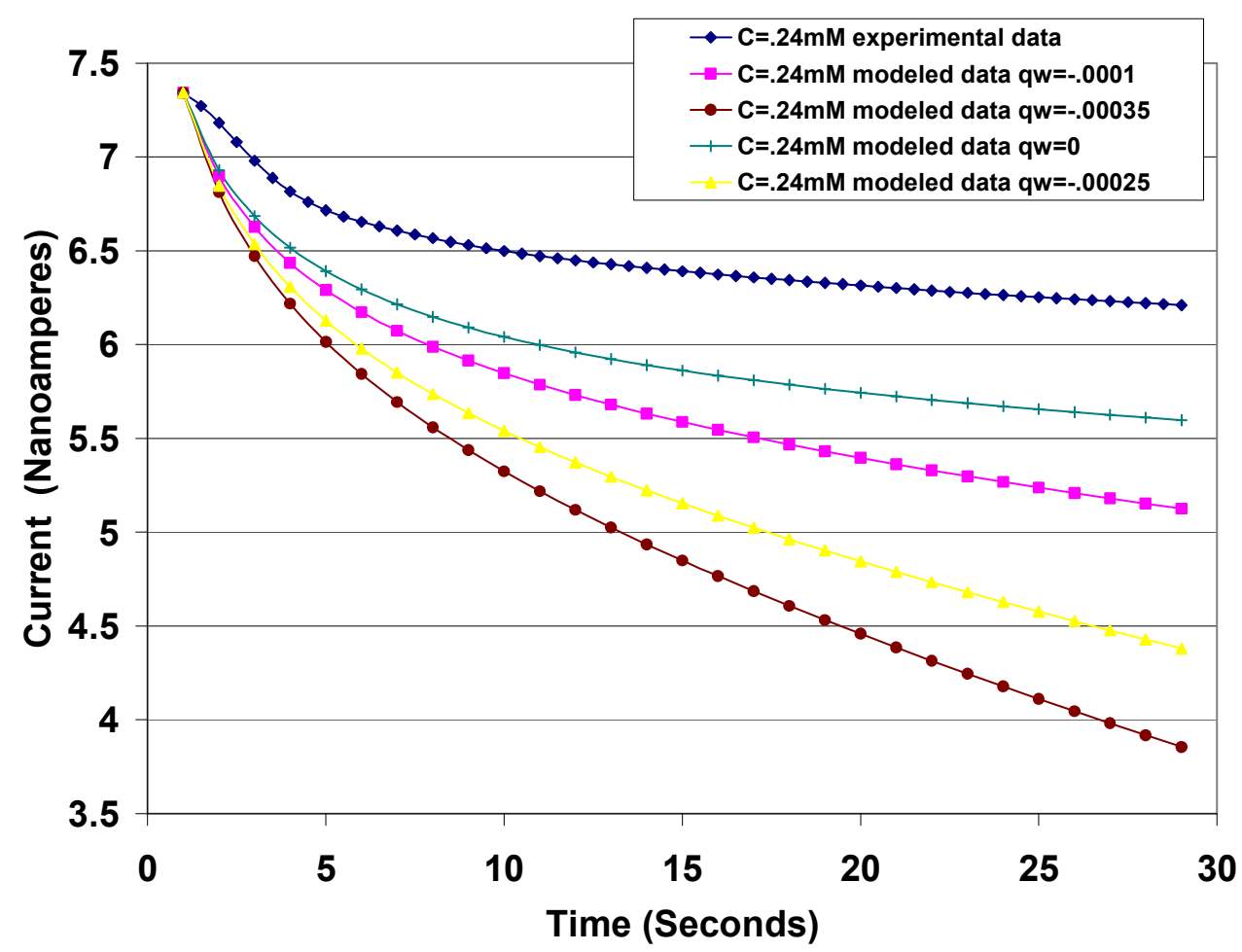

Figure 34: Comparing the experimental data and modeled data for the effects of varying Cell uptake rates (qw in $\mathrm{mM} / \mathrm{secm}^{2}$ ).

Figure 34 also confirms that the model is not perfect. Note that the model signal for the case of no cells is smaller in magnitude than the experimental case with cells present. This discrepancy suggests that the problem exists in the sensor modeling and not any other boundary condition. The next chapter discusses some of the future work planned to work around or through the sensor modeling problem. 


\section{CHAPTER V}

\section{CONCLUSIONS}

A computational fluid dynamic (CFD) model of the microphysiometer was created using both CFD-ACE and FLUENT. The properties of the oxygen sensor in the microphysiometer model have been summarized. The flow characteristics of the microphysiometer in both phases of experimental procedure, i.e. in both steady and unsteady flow have been studied.

The microphysiometer model has also been refined to take into account all the physical characteristics of the experimental setup. The microphysiometer was modeled under various conditions. From the steady state solutions the velocity fields of the flow in the chamber have been calculated, which were then used as an initial condition for the transient part of the solution. The effect of varying initial conditions in the inlet concentrations has been examined. The model shows that the initial condition on concentration only affects the total sensor output (effect of cells + sensor), and does not have any effect on the sensor output from the cells alone (section 4.3).

The cell uptake was also varied to study its effect on the sensor output. It was found that the cell uptake affected both the total sensor output and the sensor output for the cell alone. A mathematical model was created to predict the output for varying cell uptake (qw) without the use of any additional simulations (section 4.3). 
Studies have also been done to mimic the velocity profile of the inlet up to a few seconds after shutoff by using exponentially decaying inlet velocities. It was found that the exponentially decaying inlet velocities only affected the first few seconds of the total sensor output. The effects of various different exponentially decreasing inlet velocities have also been studied. The exponentially decreasing inlet velocity trends did change the shape of the curve initially but did not have any further effect on the transient solution (section 4.4).

The effects of two different types of sensors were also studied - the oxygen sensor and glucose sensor. The oxygen sensor which does not rely on any surface reaction but on a constant boundary condition $(\mathrm{C}=0 \mathrm{mM})$ at the surface of the sensor. The computational model for the glucose sensor has also been created which relies on a surface boundary reaction using Michaelis-Menten kinetics to simulate the action of the sensor at the boundary. The user-subroutine (Appendix A) required to confine the reaction to the sensor volume was also created. More detailed studies have to be done once some constants that define the rate reactions in the enzyme reaction are found (section 3.4).

Looking at the modeling results the most important thing that the modeling has determined is that the initial concentration of the micro fluidic chamber does not affect the current output at the sensor provided it is taken in relation with the signal associated with the cells off at the same initial concentration. This means that to get accurate results the experimental group should have one data set for cells uptake $=0 \mathrm{mM} / \mathrm{secm}^{2}$ for all experimental runs. This is an important observation because till now it has been difficult to calculate the initial concentration very 
accurately. The modeling indicates that this inaccuracy is not affecting the output of the sensor (but cells alone).

The parametric study for cell flux uptakes (qw) and initial cell concentration (C) has together helped in creating an equation that now predicts very accurately what the sensor output for any particular cell flux uptake and initial concentration is.

The modeled results were also compared with the experimental data. The model results are found to be within the same order of magnitude as the experimental results. It was seen that though the basic shape of the curves matched, there seemed to be some fundamental changes still required to get the magnitudes to match more accurately. This can be especially seen by the fact that when qw=0 (cells off data) the magnitude of the modeled data still differs from the experimental data. It was also seen that when comparing total current, i.e. the effect of cells as well as sensor, the output is affected to a much greater extent by the cell flux than by initial concentration (section 4.4).

From the FLUENT model it was calculated that the highest value of cell uptake that can be taken without negative values being seen in the steady state solution is $-2.5 \mathrm{E}-{ }^{5} \mathrm{mM} / \mathrm{secm}^{2}$.

\subsection{Future work}

From the comparisons between the modeled data and the experimental data it is seen that to make the model more accurate, methods have to be found to slow down the amount of analytes being eaten up by the sensor. This means that the basic ideal sensor theory that the sensor eats all the analytes that reaches it, will have to be 
modified. A delay will have to be incorporated into the model which will force the rate at which the analytes are being eaten up by the sensor to slow down.

There are a few methods by which this can be done. One of the methods involves trying to model the characteristics of an electrode more closely. This means that electrochemical modeling needs to be incorporated into the model. The problem here is that the geometry then will become very complex similar to the glucose model. The electrode shape will have to be very accurate and porous walls need to be used to model some parts of the electrode surface. Also the electrochemical modeling would require a number of rate reactions factors that are not readily available. This means that a trial and error parametric sweep would be required and this would take a very long time to model.

Another method is to try and slow down the rate of cell uptake by the use of simpler boundary conditions. One way is to modify the sensor wall boundary condition to some other value that is close to but not equal to $0 \mathrm{mM} \cdot \mathrm{sec} / \mathrm{m}^{2}$. Also a system of boundary conditions that divide the initial area of the sensor surface into various parts some of which have zero boundary condition and some that do not can be used to try and slow down the cell uptake.

The factors that are yet to be considered include the assumption that the cell uptake never changes with respect to time. This is a factor for which not much experimental information is available. It will be worthwhile to study a few cases where the cell uptake increases with time, and a few cases where it decreases with time to see how this affects the sensor output. The next step would be to see what 
type of increase or decrease in cell uptake most closely matches the experimental data.

In parametric studies another thing to look at is the concentration of the cells at the bottom surface of the microphysiometer. Right now the assumption is that the cells are distributed uniformly across the bottom surface. This is not necessarily true. During experimental cell culture it is possible for the cells to grow unevenly. The effect the uneven concentration of cells at the bottom surface of the microphysiometer will have on the current output of the sensor will also have to be studied.

The computational model provides useful predictions of trends, but further development needs to be done in order to accurately predict the magnitude of the actual sensor signals. 


\section{APPENDIX A}

\section{User Subroutine in CFDACE for glucose sensor}

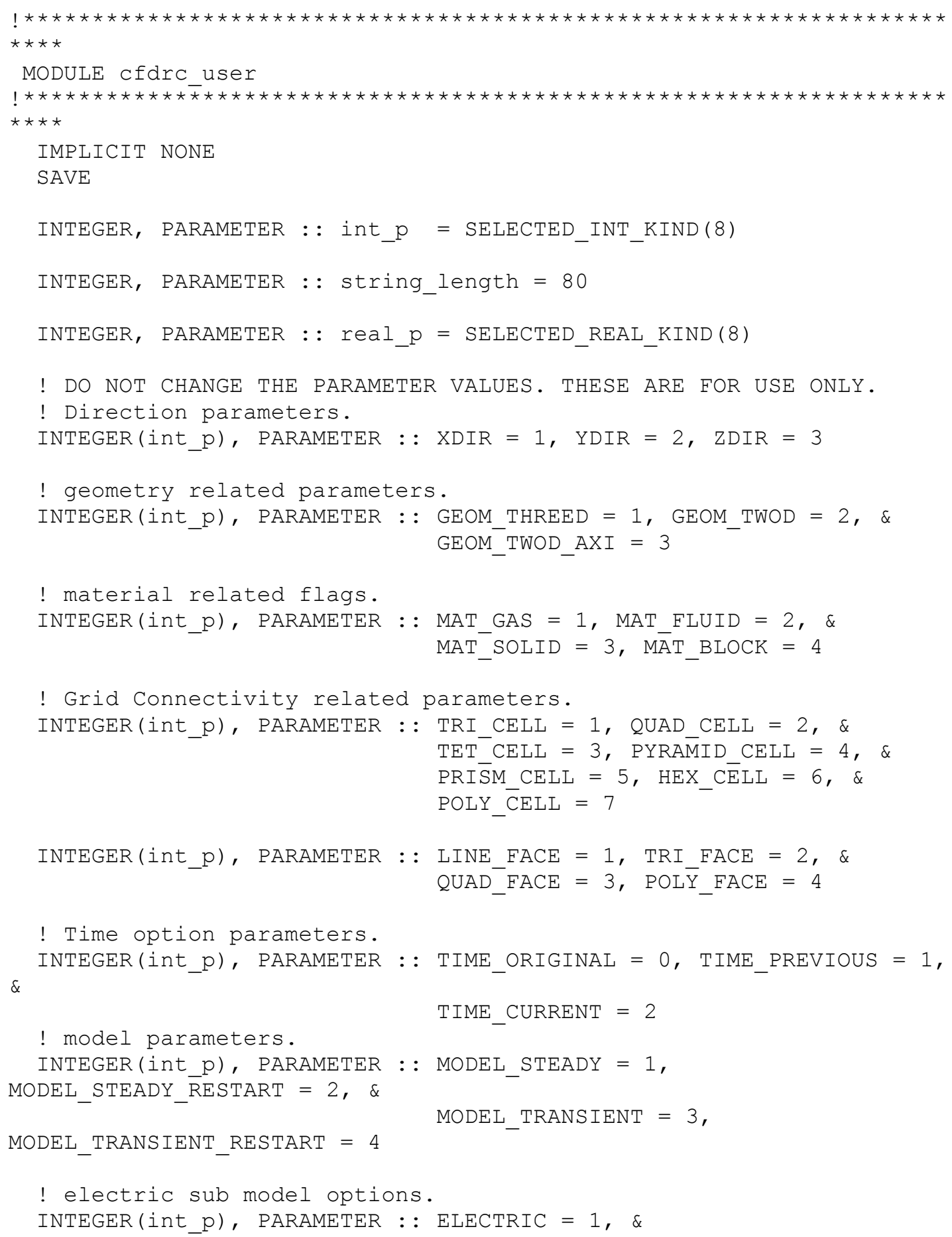




$$
\begin{aligned}
& \text { ELECTRIC_ELECTROSTATICS_FVM }=2, \& \\
& \text { ELECTRIC_ELECTROSTATICS_BEM }=3, \& \\
& \text { ELECTRIC_DC_CONDUCTION }=4, \& \\
& \text { ELECTRIC_AC_CONDUCTION }=5, \& \\
& \text { ELECTRIC_TIME_DOMAIN }=6
\end{aligned}
$$

! global bc types.

INTEGER (int_p), PARAMETER : : BC_TYPE_INLET $=1$, $\&$

BC_TYPE_WALL $=2, \&$

$\mathrm{BC}^{-}$TYPE_EXIT $=3, \&$

$\mathrm{BC}^{-}$TYPE_INTERFACE $=4, \&$

${ }^{-}{ }^{-}$TYPE ${ }^{-}$F_F_INTERFACE $=5, \&$

$\mathrm{BC}^{-}$TYPE ${ }^{-}{ }^{-}{ }^{-}$INTERFACE $=6, \&$

$\mathrm{BC}^{-}{ }^{-} \mathrm{TYPE} \mathrm{S}^{-} \mathrm{F}^{-}$INTERFACE $=7, \&$

$\mathrm{BC}^{-}$TYPE ${ }^{-}{ }^{-}$INTERFACE $=8, \&$

BC TYPE_B_B INTEREACE $=9, \approx$

$\mathrm{BC}^{-}{ }^{-} \mathrm{TYPE}{ }^{-} \mathrm{S}^{-}{ }^{-}$INTERFACE $=10, \&$

$\mathrm{BC}^{-}{ }^{-} \mathrm{TYPE}{ }^{-} \overline{\mathrm{Y}} \overline{\mathrm{MM}}=11, \&$

$\mathrm{BC}^{-}$TYPE_CYCLIC $=12, \&$

BC_TYPE_THINWALL $=13$

! heat transfer bc subtypes

INTEGER (int p), PARAMETER : : BC HEAT INOUT $=1, \varepsilon$

$\mathrm{BC}^{-} \mathrm{HEAT}^{-} \mathrm{SYMM}=2, \&$

$\mathrm{BC}^{-} \mathrm{HEAT}^{-}$ISOTHERMAL $=3, \&$

BC_HEAT_ADIABATIC $=4, \&$

$\mathrm{BC}^{-} \mathrm{HEAT}^{-} \mathrm{FIX} \mathrm{Q}=5, \&$

$\mathrm{BC}^{-} \mathrm{HEAT}{ }^{-} \mathrm{NEWTON}=6, \&$

$\mathrm{BC}^{-}{ }^{-} \mathrm{HEAT}^{-} \mathrm{EXT}$ RADIATION $=7, \&$

BC_HEAT_CONJUGATE_INTERFACE $=8, \&$

BC_HEAT $^{-}$THINWALL $=9, \&$

$\mathrm{BC}^{-} \mathrm{HEAT}^{-} \mathrm{CYCLIC}=10, \&$

$\mathrm{BC}^{-} \mathrm{HEAT}^{-}$INTERFACE $=11, \&$

$\mathrm{BC}^{-}{ }^{-} \mathrm{HEAT}^{-}{ }^{-}$MIXING PLANE $=12, \&$

$\mathrm{BC}^{-} \mathrm{HEAT}^{-} \mathrm{COUPLE}{ }^{-}=13, \&$

$\mathrm{BC}_{-}^{-} \mathrm{HEAT}$-CHIMERA $=14$

! flow bc subtypes

INTEGER (int_p), PARAMETER : : BC_FLOW_FIXM_INLET $=1$,

$\mathrm{BC}^{-}$FLOW ${ }^{-}$FIXP OUTLET $=2, \&$

$\mathrm{BC}^{-} \mathrm{FLOW}^{-} \mathrm{WALL}^{-}=3, \&$

$\mathrm{BC}^{-} \mathrm{FLOW}{ }^{-} \mathrm{SYMM}=4, \&$

$\mathrm{BC}^{-}$FLOW ${ }^{-}$FIXP_EXTRAPOLAT_OUTLET $=5, \quad$

$\mathrm{BC}^{-}$FLOW ${ }^{-}$FIXPT INLET $=6, \&$

$\mathrm{BC}^{-}$FLOW ${ }^{-}$FIXP $\overline{\mathrm{INLET}}=7, \quad$,

$\mathrm{BC}^{-} \mathrm{FLOW}{ }^{-} \mathrm{CYCL} \overline{\mathrm{I}} \mathrm{C}=8, \&$

$\mathrm{BC}^{-}$FLOW ${ }^{-}$INTERFACE $=9, \&$

BC_FLOW_MIXING_PLANE $=10, \&$

$\mathrm{BC}^{-} \mathrm{FLOW}{ }^{-} \mathrm{COUPLE}{ }^{-}=11, \&$

$\mathrm{BC}^{-} \mathrm{FLOW}{ }_{-}^{-} \mathrm{CHIMERA}=12$

! electric bc subtypes.

INTEGER(int_p), PARAMETER : : BC_ELECTRIC_FIX_POTENTIAL = 1, \&

$\mathrm{BC}^{-}$ELECTRIC FIX FLUX $=2, \&$

$\mathrm{BC}^{-} \mathrm{ELECTRIC}{ }^{-} \mathrm{SYMM}_{\bar{M}}=3, \&$

$\mathrm{BC}^{-}$ELECTRIC_CYCLIC $=4, \&$ 


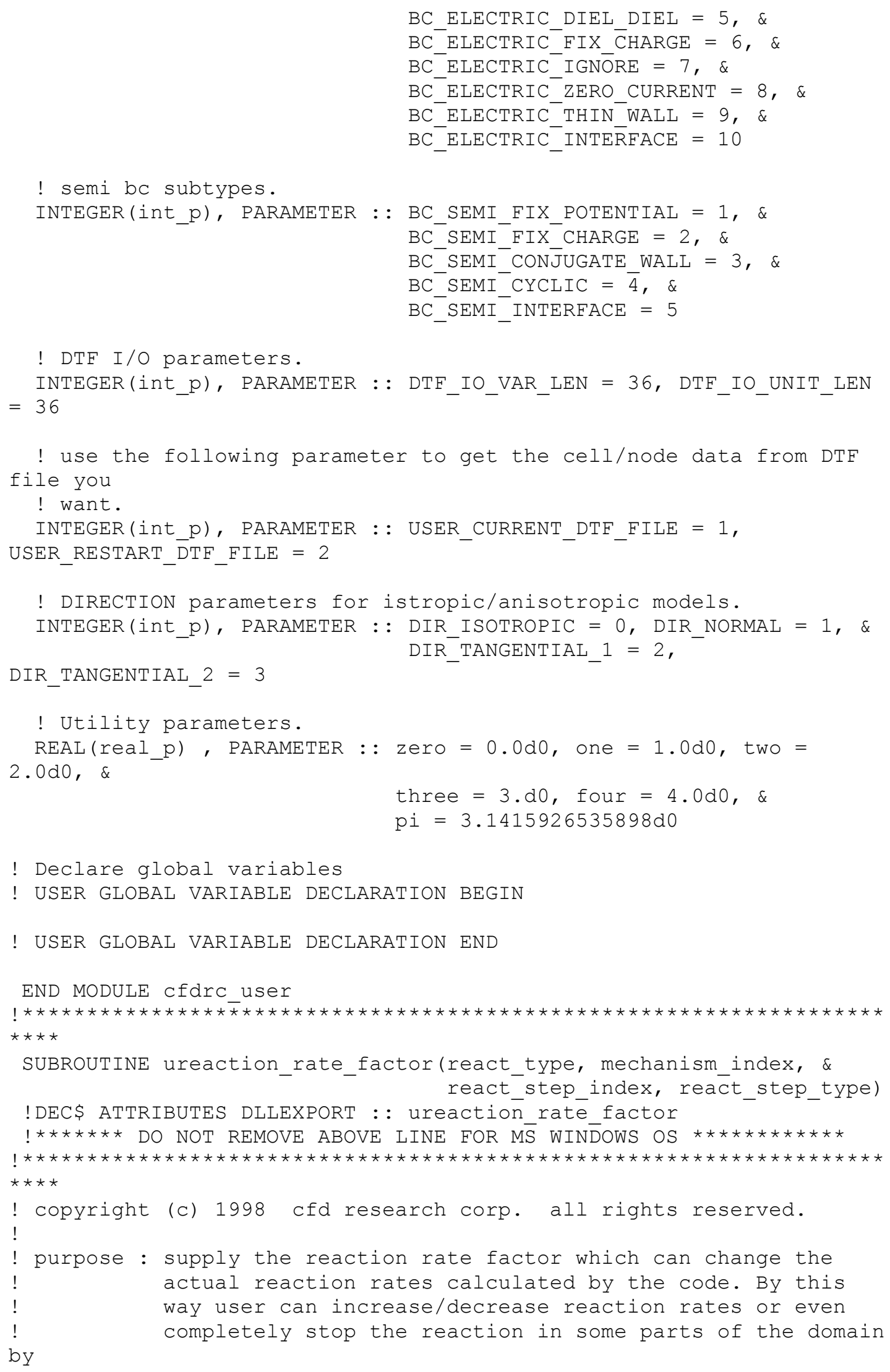




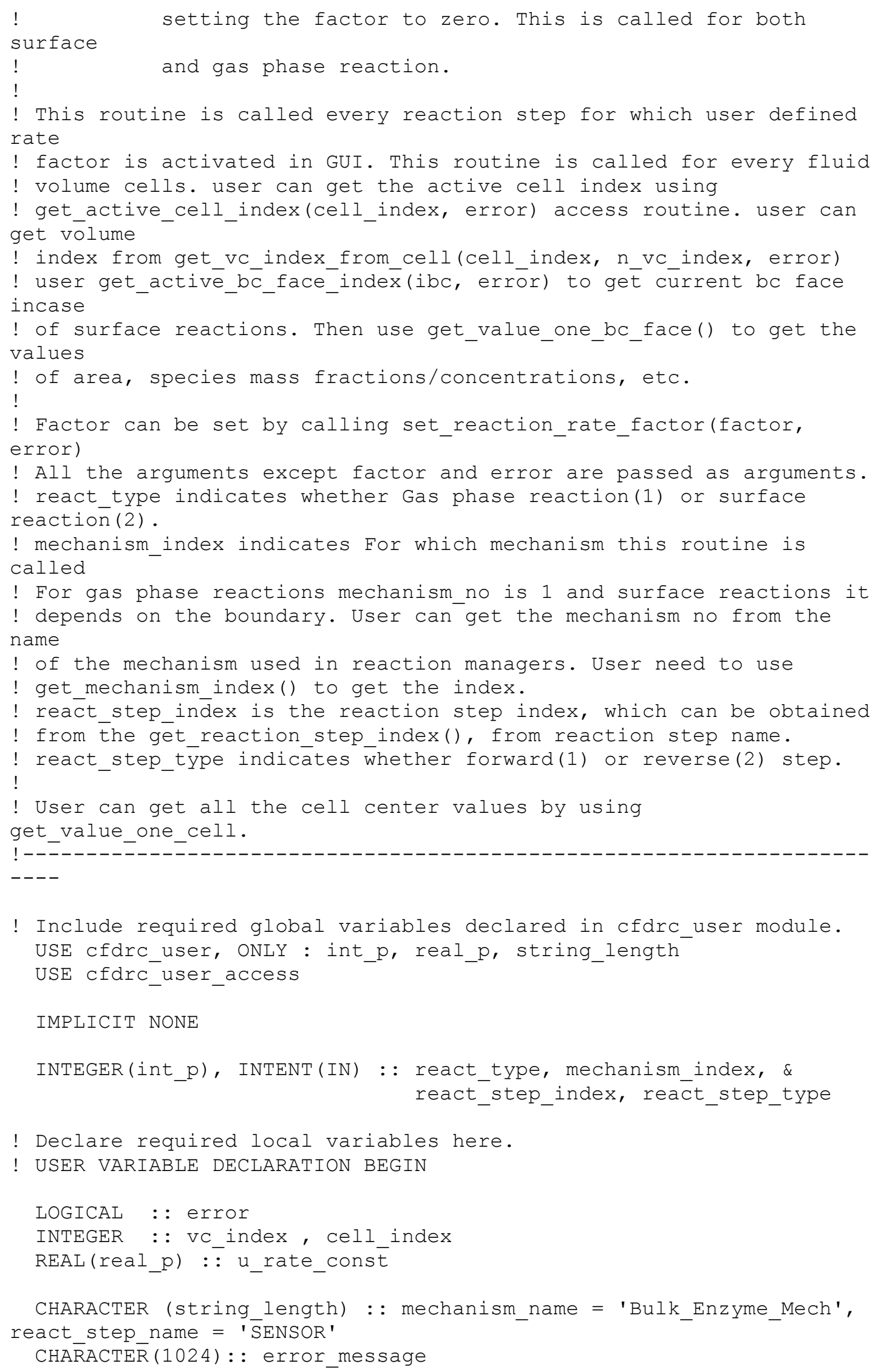




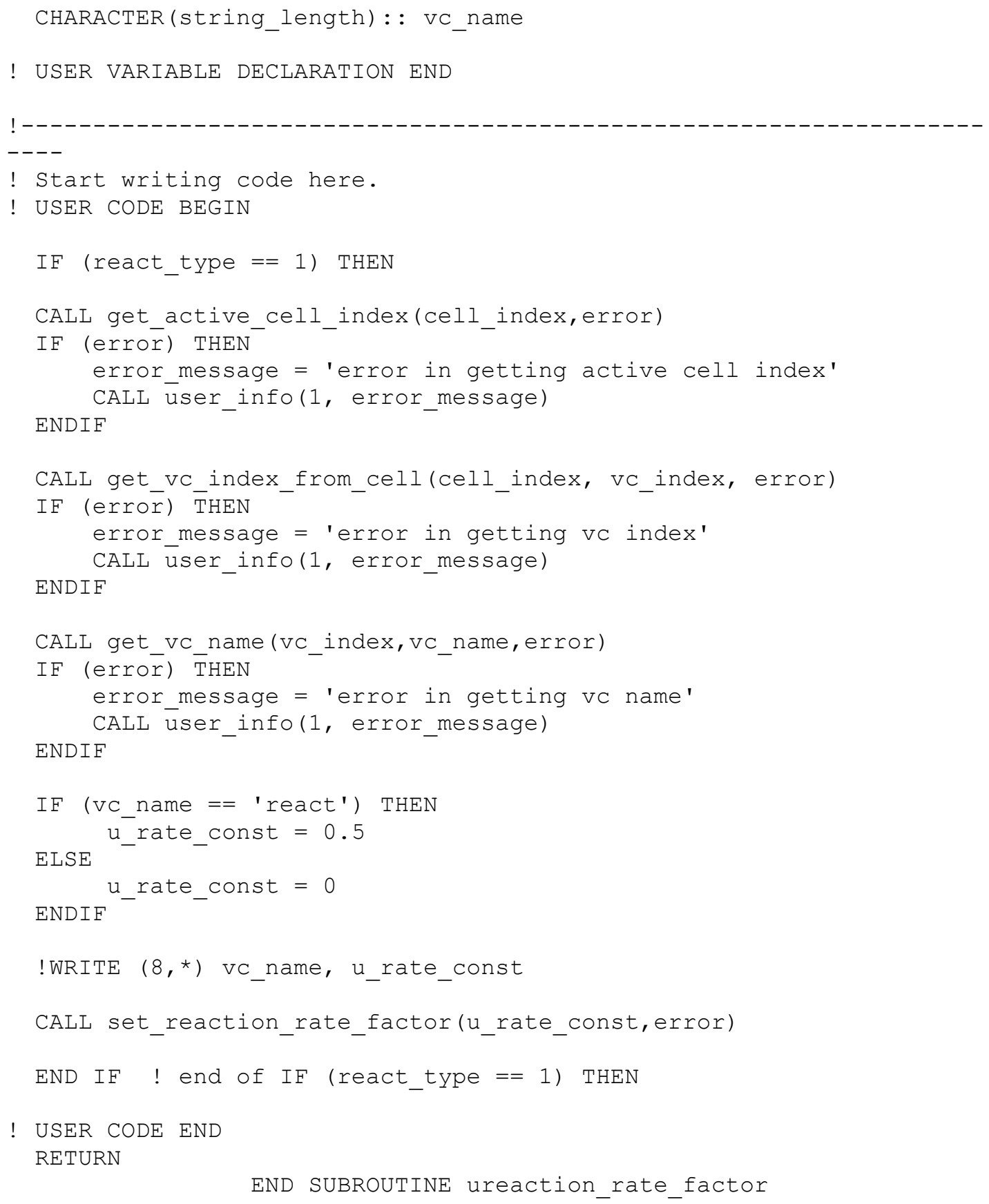




\section{REFERENCES}

Aravanis, Alexander M., Debusschere, B.Derek and Chruscinski, Andrzej J. (2001), “ A Genetically Engineered Cell-based Biosensor for Functional Classification of Agents," Biosensors \& Bioelectronics 16, pp. 571-577.

Abbott, M.B., and Basco, D.R. (1989), Computational Fluid Dynamics - an Introduction for Engineers, Longman Scientific \& Technical Publication.

Alexandrou Andreas (2001), Principles of Fluid Mechanics, Prentice Hall Publication

Astrup P.B (ed) 1986, History of blood gas analysis. IV., "Leland Clark's oxygen electrode", Journal Clin. Monit. April 2(2), pp. 125-139.

Becker, Wayne M., Reece, Jane B., and Poenie, Martin F. (1996), The World of the Cell, The Benjamin/Cummings Publishing Company.

Bott, Adrian W. (1998), "Electochemical Methods for the Determination of glucose," Current Separation 17:1, pp.25-31.

Bruce, Alberts, Bray, Dennis, Johnson, Alexander, et al. (1998), Essential Cell Biology: An Introduction to the Molecular Biology of the Cell, Taylor \& Francis Publishing Goup.

Buerk, Donald G. (1993), Biosensors - Theory and Apllications, Technomic Publishing Company.

Burns, Salme DeAnna, and Bau, Haim (2000), "Fabrication of A Call-based Biosensor using Green Tape Ceramics with Human Embryonic Kidney and Sea Urchin Egg Cells."

Clark, L.C., and Lyons, C. (1962), "Electrode Systems for Continous Monitoring in Vascular Surgery," Ann.N.Y.Acad.Sci 102, pp.29-45.

Cunningham, Alice J. (1998), Introduction to Bioanalytical Sensors, John Wilely \& Sons.

Eklund S.E., Taylor D., Kozlov E., Prokop A., Cliffel E.D.,(2004), “A Microphysiometer for Simultaneous Measurement of Changes in Extracellular Glucose, Lactate, Oxygen, and Acidification Rate.”, Anal. Chem, 76(3), pp.519-527.

Fick, A. (1885), Ann. Physik, Leipzig 170, pp. 59.

Fraser, D.M. (1994), "Biosensors: Making sense of them,” Medical Device Technology Vol.5, No.8, pp. 38-41. 
Fraser, D.M. (1995), “Biosensor Markets- Oppertunities and Obstacles” Medical Device Technology, Vol.6, No.2, pp.32-37.

Fraser, D.M. (1994), "Glucose biosensors - the sweet smell of success”, Medical Device Technology, Vol.5, No.9, pp.44-47, 1994.

George M.W., Abraham D.S., “Flexible Methods for Microfluidics”, Vol.54, No.6, pp42.

Higson S.P.J, Reddy S.M., and Vadgama P.M., "Enzyme and other biosensors : Evolution of a technology", Engineering Science and Education Journal, Feb 1994, pp.41-48.

Janata, J., "Principles of chemical sensors", Plenum Press, (1989).

Kissinger, Peter T. (1997), "Introduction to Amperometric Biosensor Configurations," Current Separations Vol. 16 No. 3.

Kruger, Jan, Singh, Kirat, O’Neill, Alan, Jackson, Carl, Morrison, Alan, O’Brien, Peter (2002), "Development of a Microfluidic Device for Fluorescence Activated Cell Sorting," J.Micromech. Microeng. 12, pp. 486-494.

Mohanty, Saraju P. (2001), "Biosensors: A Survey Report.” www.csee.usf.edu/ $\sim$ smohanty/research

User's Manual, Cytosensors Microphysiometer, Molecular devices Incorporated.

Verhaegen, Katarina, Baert, Kris, Simaels, Jeannine, Driessche, Willy Van (2000), “ A High-Throughput Silicon Microphysiometer," Sensors and Actuators 82, pp.186190.

White, Frank M., "Fluid Mechanics,” McGraw-Hill, (1994).

Whiteley, Jonathan P., Gavaghan, David J., Hahn, Clive E.W. (2001), "Some Factors Affecting Oxygen Uptake by Red Blood Cells in the Pulmonary Capillaries," Mathematical Biosciences 169, pp.153-172.

Wu Yicong, Wang Ping, Ye Xuesong, Zhang Gaoyan, et al. (2001), "Drug Evaluations using a Novel Microphysiometer based on cell-based Biosensors," Sensors and Actuators B 80, pp.215-221. 
